(Supporting Information)

\title{
Self-Assembly-Driven Nanomechanics in Porous Covalent Organic Framework Thin Films
}

Kaushik Dey, ${ }^{1}$ Surojit Bhunia, ${ }^{1,2}$ Himadri Sekhar Sasmal, ${ }^{1}$ C. Malla Reddy ${ }^{1,2}$ and Rahul Banerjee ${ }^{1,2 *}$

${ }^{1}$ Department of Chemical Sciences, Indian Institute of Science Education and Research, Kolkata, Mohanpur 741246, India.

${ }^{2}$ Centre for Advanced Functional Materials, Indian Institute of Science Education and Research, Kolkata, Mohanpur 741246, India.

*Email: r.banerjee@iiserkol.ac.in; Tel: +033-6136-0000-1327.

cmallareddy@gmail.com; Tel: +033-6136-0000-1262. 
Table of Contents

\begin{tabular}{|c|l|c|}
\hline Section & & Page No \\
\hline S-1 & Materials and Instrumentation & S3 \\
\hline S-2 & Synthetic procedures & S5 \\
\hline S-3 & PXRD Structure Refinement & S7 \\
\hline S-4 & FTIR spectra & S9 \\
\hline S-5 & TG analyses & S9 \\
\hline S-6 & Nitrogen adsorption analysis & S10 \\
\hline S-7 & SEM and TEM analyses & S11 \\
\hline S-8 & AFM analyses & S13 \\
\hline S-9 & DLS analyses & S15 \\
\hline S-10 & Nanoindentation experiments & S16 \\
\hline S-11 & Additional data & S34 \\
\hline S-12 & References & \\
\hline
\end{tabular}




\section{Section S-1: Materials and Instrumentation}

\section{Materials}

The starting materials 1,3,5-triformylphloroglucinol (Tp) was synthesized in the lab following the previous literature protocols. ${ }^{1}$ All commercially available reagents and solvents were used without further purification. All the commercially available materials were bought from Sigma-Aldrich, TCI chemicals, Avra chemicals and Fisher Scientific depending upon their availability.

\section{General instrumentations and methods}

Powder X-ray diffraction (PXRD) data were collected using a Rigaku, SmartLab with high-intensity rotating anode X-ray generator. All the COFs were recorded in the $2 \theta$ range between $2-40^{\circ}$ and data was collected with the help of Control Win software. The radiation used was $\mathrm{Cu} \mathrm{K} \alpha(\lambda=1.54 \AA)$ with a Ni filter, and the data collection was carried out using an Aluminium holder at a scan speed of $1^{\circ} \mathrm{min}^{-1}$ and a step size of $0.02^{\circ}$. To calculate the unit cell parameters, two possible stacking models (eclipsed AA and staggered AB) were built using Accelrys, Material Studio-6 software using the Self-Consistent Charge Density Functional Tight-Binding (SCC-DFTB) method (J. Am. Chem. Soc. 2012, 134, 19524; J. Am. Chem. Soc. 2013, 135, 17853). Several stacking possibilities were considered for reasons reported in the literature.

Fourier transform infrared (FT-IR) spectra were obtained using a Bruker Optics ALPHAE spectrometer with a universal Zn-Se ATR (attenuated total reflection) accessory. FT-IR data are reported with a wave number $\left(\mathrm{cm}^{-1}\right)$ scale.

Thermogravimetric analyses (TGA) were carried out on a TG50 analyzer (Mettler-Toledo) and a SDT Q600 TG-DTA analyzer under $\mathrm{N}_{2}$ atmosphere at a heating rate of $10{ }^{\circ} \mathrm{C} \mathrm{min}{ }^{-1}$ within a temperature range of $30-900{ }^{\circ} \mathrm{C}$.

Scanning Electron Microscopy (SEM) measurements were executed with a Zeiss SUPRA 55 VP scanning electron microscope and Zeiss SIGMA Scanning Electron Microscope equipped with tungsten filament as electron source operated at $10 \mathrm{kV}$. The samples were prepared simply by putting a drop of COFs dispersed in isopropanol on a clean piece of 
Silicon wafer. To avoid charging during SEM analyses, we coated all the COFs samples with a thin layer of gold by a SCD 040 Balzers Union prior to analyses.

Transmission Electron Microscopy (TEM) analyses were performed using a UHR-FEGTEM, DST-FIST facility of IISER Kolkata at an accelerating voltage of $200 \mathrm{kV}$. The TEM Samples were prepared for analyses by drop casting the samples (dispersed in isopropanol) on copper grids TEM Window (TED PELLA, INC. 200 mesh).

$\mathbf{N}_{2}$ adsorption analyses were performed at $77 \mathrm{~K}$ using a liquid nitrogen bath $(77 \mathrm{~K})$ on a Quantachrome Quadrasorb automatic volumetric instrument. All the COFs samples were outgassed for $12 \mathrm{~h}$ at $120{ }^{\circ} \mathrm{C}$ under vacuum prior to the gas adsorption studies. The surface areas were evaluated using Brunauer-Emmett-Teller (BET) model applied between $\mathrm{P} / \mathrm{P}_{0}$ values of 0.05 and 0.3 for mesoporous COFs. The pore size distributions were calculated using the non-localized density functional theory (NLDFT) method. The surface area of each of the COF has been measured multiple times and then averaged out for proper comparison.

Atomic Force Microscopy (AFM) imaging technique was used with Asylum AFM AC 240 TS-R3 silicon cantilever probes. Topographic images, amplitude of the samples was obtained and analyzed by standard AC mode imaging.

Confocal laser scanning microscope (CLSM) (Zeiss, Oberkochen, Germany Model LSM 710) was used to achieve background free high-resolution images of micro porous COFfoam. The confocal microscope was equipped with EC Epiplan-APOCHROMAT objective of 50X magnification and a numerical aperture of 0.9. Fluorescence from the sample was excited with the 488-nm line of an Argon laser. The fluorescence was separated from the excitation light by band pass sliders $(493 \mathrm{~nm}-619 \mathrm{~nm})$ in front of spectral detectors. The microscope objective was attached to an opto-electronically coded focus z-driver to scan the objective along the vertical $z$ direction and produce three dimensional images. A pinhole was used to reject the out-of-plane light for background rejection hence better signal to noise ratio. We typically acquired 150-200 sliced stacks of 512 X 512 pixel images in the horizontal $x-y$ plain separated by $0.25 \mu \mathrm{m}$ in the $z$ direction. The length of the full-sized field of view is approximately $283 \mathrm{um}$ and a depth approximately $40 \mu \mathrm{m}$. The images were processed using the Zen software (Zeiss, Oberkochen). 


\section{Section S-2: Synthetic Procedures and methods}
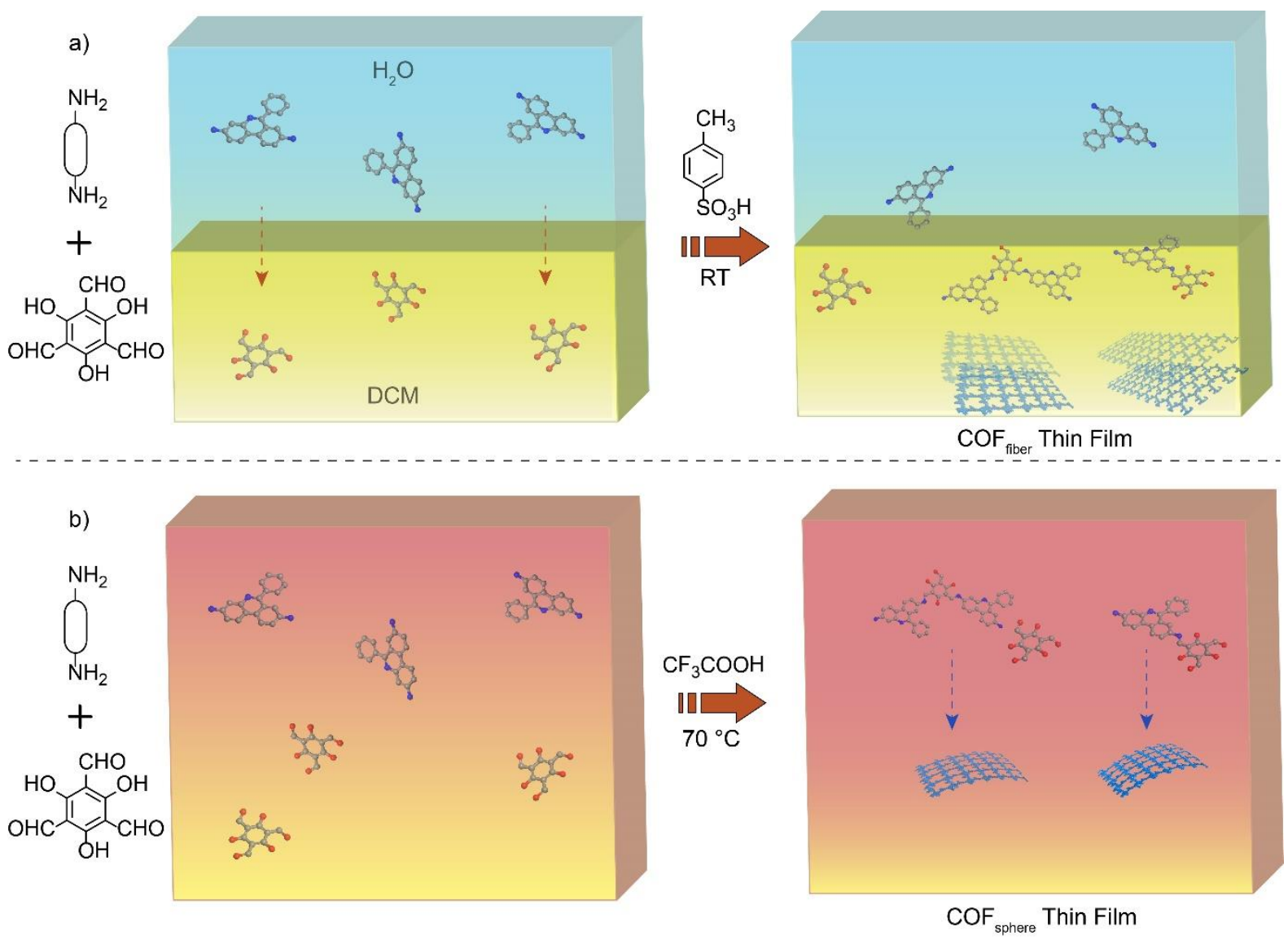

Figure S1 | Synthesis of COF-thin films. a) $\mathrm{COF}_{\text {fiber }}$ and b) $\mathrm{COF}_{\text {sphere }}$ films.

\section{Synthesis of COF-thin films.}

a) $\mathrm{COF}$ fiber film: Tp-Azofiber, and $\mathrm{Tp}-\mathrm{DPP}_{\text {fiber }}$ films were synthesized in a glass beaker (opensystem). First, $0.075 \mathrm{mmol}$ of 1,3,5-triformylphloroglucinol (Tp) (15.7 mg) dissolved in 100 $\mathrm{ml}$ of dichloromethane was poured into the beaker. A spacer layer of $60 \mathrm{ml}$ of water was added on top of the aldehyde solution. Finally, $0.112 \mathrm{mmol}$ of diamine [4,4'-azodianiline (Azo), $23.7 \mathrm{mg}$; 3,8-Diamino-6-phenylphenanthridine (DPP), $31.9 \mathrm{mg}$ ]-PTSA (0.224 mmol, $38.5 \mathrm{mg}$ ) were dissolved in $100 \mathrm{ml}$ of water and added slowly on top of the spacer solution over a period of $30 \mathrm{~min}$. The system was kept at room temperature for $72 \mathrm{~h}$ in undisturbed condition. The thin films formed at the interface and the bottom surface of the beaker were collected by removing the top aqueous layer with a dropper and washed with water, dimethylacetamide (DMAc), and acetone to purify the COFs. To perform the nanoindentation experiments, $1 \mathrm{~cm}$ sized glass substrate were placed on the bottom of the glass beaker, where 
the $\mathrm{COF}_{\text {fiber }}$ films were crystallized. The COF coated substrates were similarly washed with water, dimethylacetamide (DMAc), acetone and utilized further.

a) $\mathrm{COF}_{\text {sphere }}$ film: $\mathrm{Tp}-\mathrm{Azo}_{\text {sphere }}$, and $\mathrm{Tp}-\mathrm{DPP}$ sphere films were synthesized in a round-bottom flux (closed-system). The diamine $(0.045 \mathrm{mmol})$ (Azo and DPP) was added into $100 \mathrm{ml}$ dry dichloromethane (DCM) at room temperature, which resulted in a transparent homogeneous solution (dark yellow and light yellow coloured transparent homogeneous solution for Azo and DPP amine respectively). Trifluoroacetic acid (20 $\mu \mathrm{l})$ was added directly to the solution as the catalyst for Schiff base reaction which turned the colour of the solution from dark yellow and light yellow to wine red and orange respectively. Finally, a solution of $0.03 \mathrm{mmol}$ trialdehyde (Tp) in $100 \mathrm{ml}$ dry DCM was added slowly with the stirring at room temperature. The reaction mixture was refluxed at $\sim 70^{\circ} \mathrm{C}$. After 36 hours, $\mathrm{COF}_{\text {sphere }}$ films were found to crystallize on the wall of the flask. The films were collected by scratching, washed with water, dimethylacetamide (DMAc), and acetone for purification. To perform the nanoindentation experiments, $1 \mathrm{~cm}$ sized glass substrate were introduced inside the RB, where the $\mathrm{COF}_{\text {sphere }}$ films were crystallized. The COF coated substrates were similarly washed with water, dimethylacetamide (DMAc), acetone and utilized further.

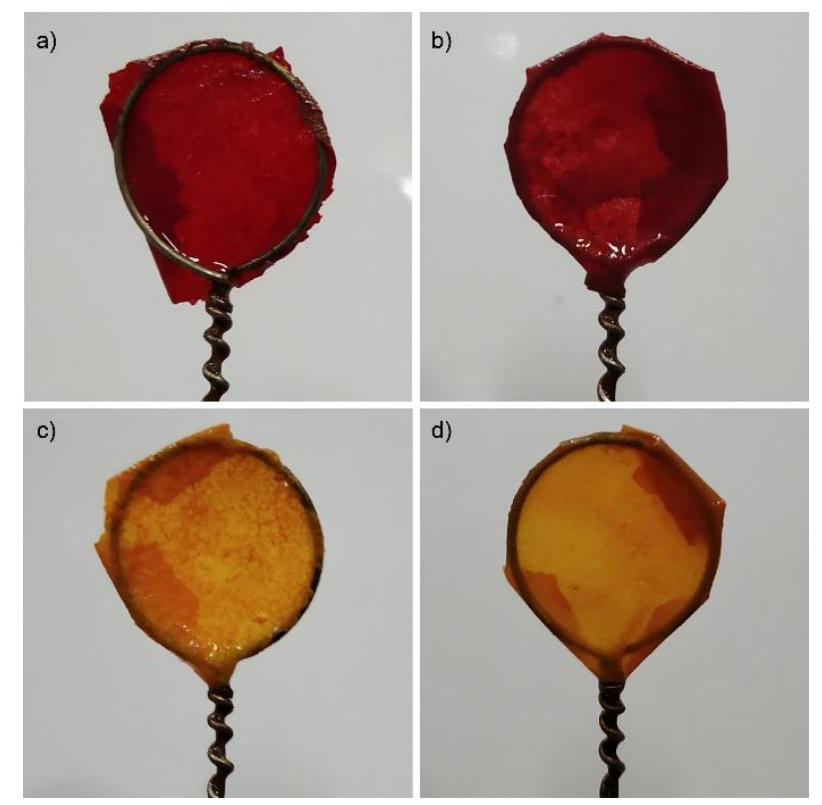

Figure S2 | Digital images of self-standing COF thin films mounted on a wire-loop. a) TpAzofiber; b) Tp-Azo sphere $_{\text {c) }}$ Tp-DPP ${ }_{\text {fiber }}$; d) Tp-DPP sphere $_{\text {thin-film. }}$ 


\section{Section S-3: PXRD Structure Refinement}

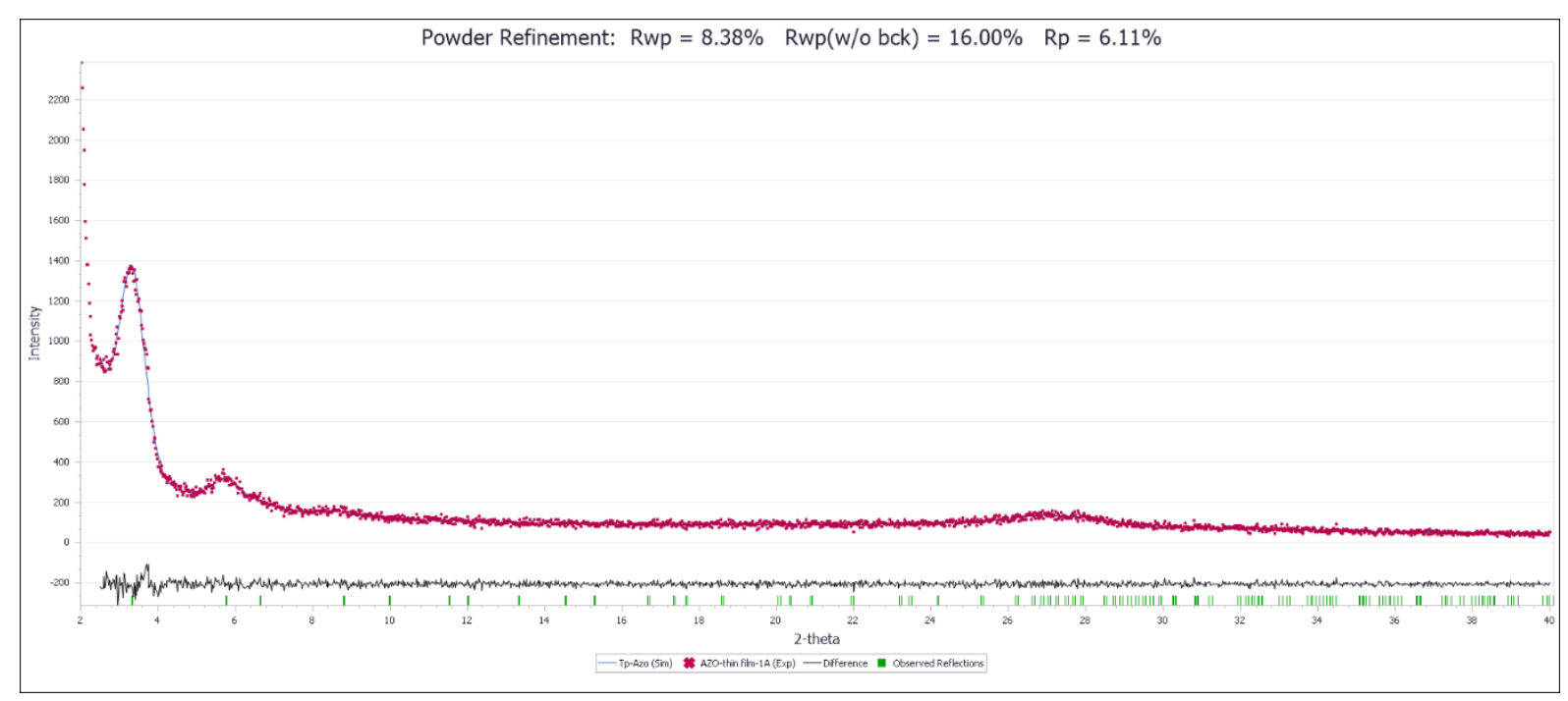

Figure S3 | Pawley refinement for Tp-Azofiber film. Pawley refinement indicates good agreement between the simulated (blue) and experimental (red) PXRD patterns.

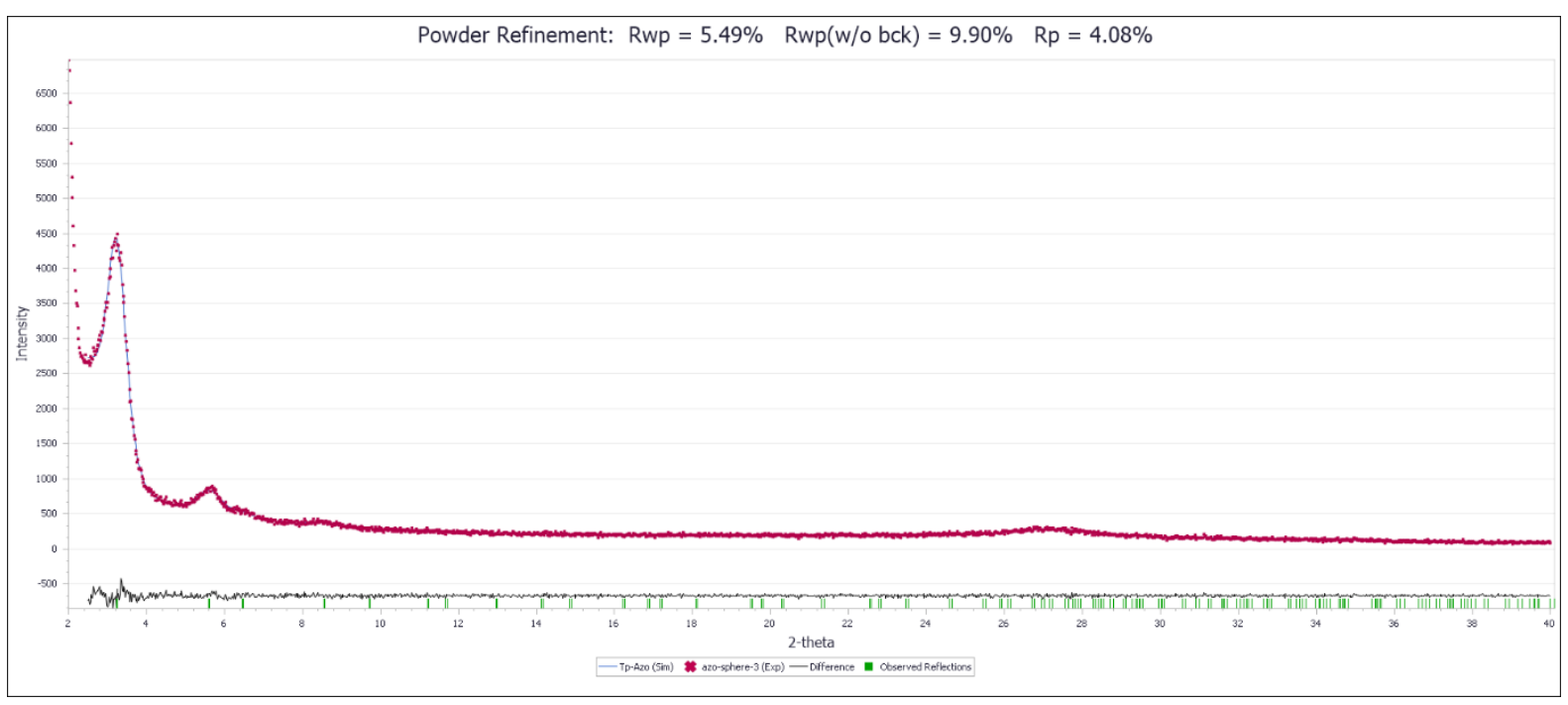

Figure S4 | Pawley refinement for Tp-Azosphere film. Pawley refinement indicates good agreement between the simulated (blue) and experimental (red) PXRD patterns. 


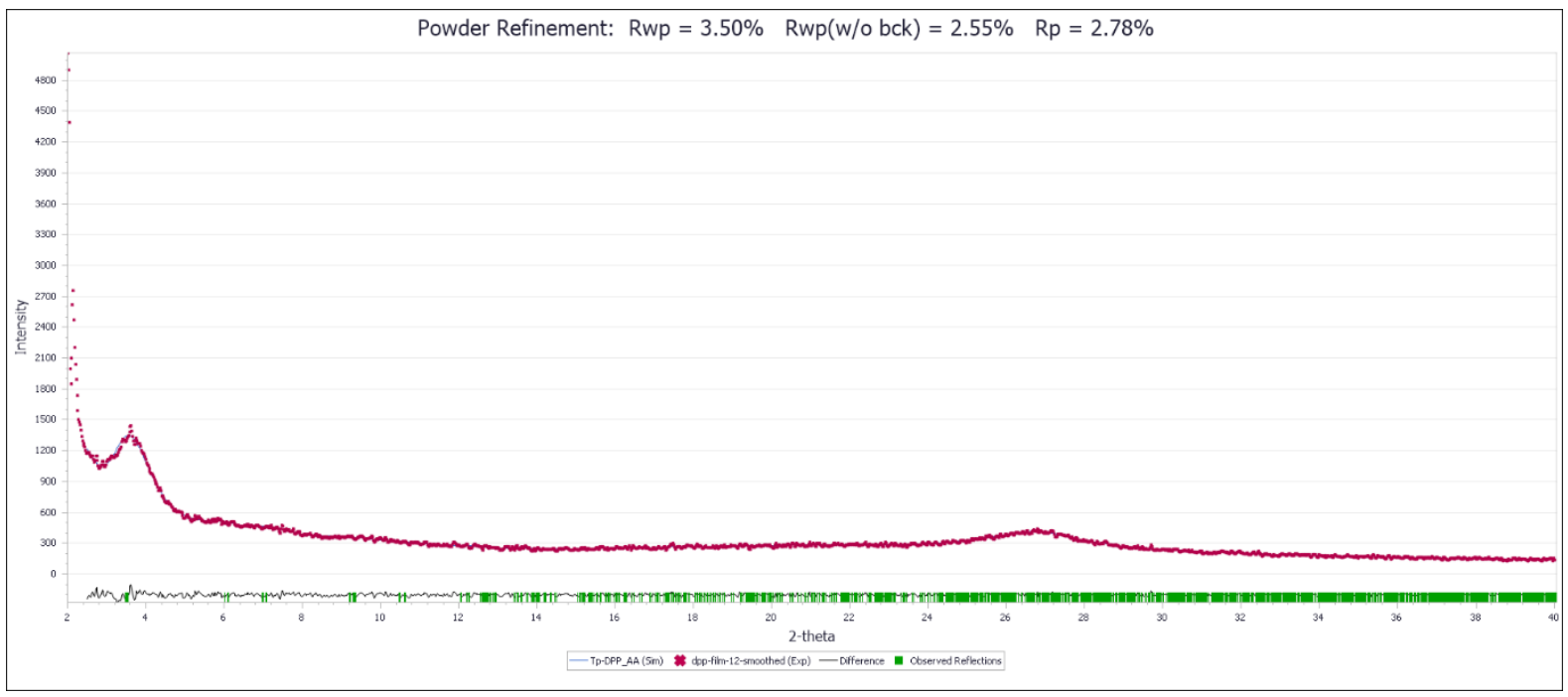

Figure S5 | Pawley refinement for Tp-DPPfiber film. Pawley refinement indicates good agreement between the simulated (blue) and experimental (red) PXRD patterns.

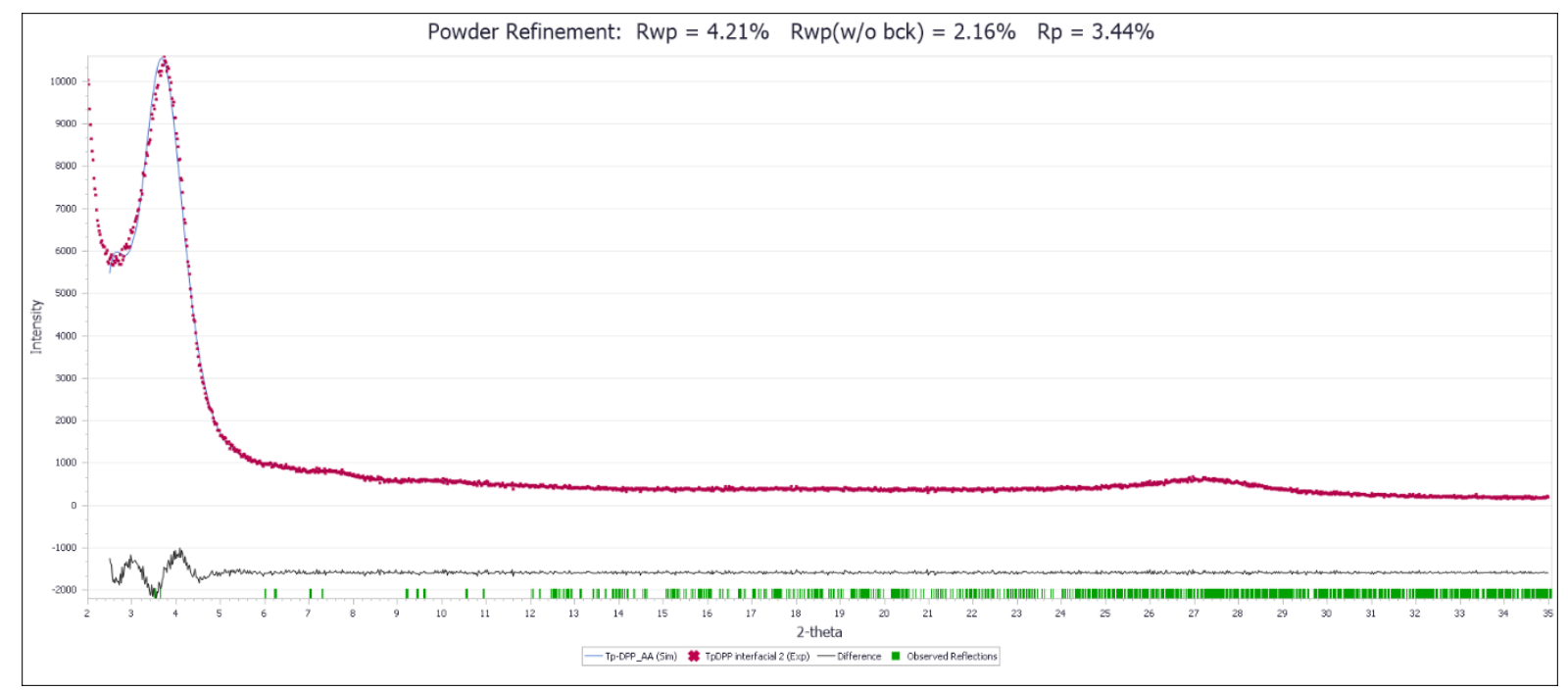

Figure S6 | Pawley refinement for Tp-DPP sphere film. Pawley refinement indicates good agreement between the simulated (blue) and experimental (red) PXRD patterns. 


\section{Section S-4: FTIR spectra}
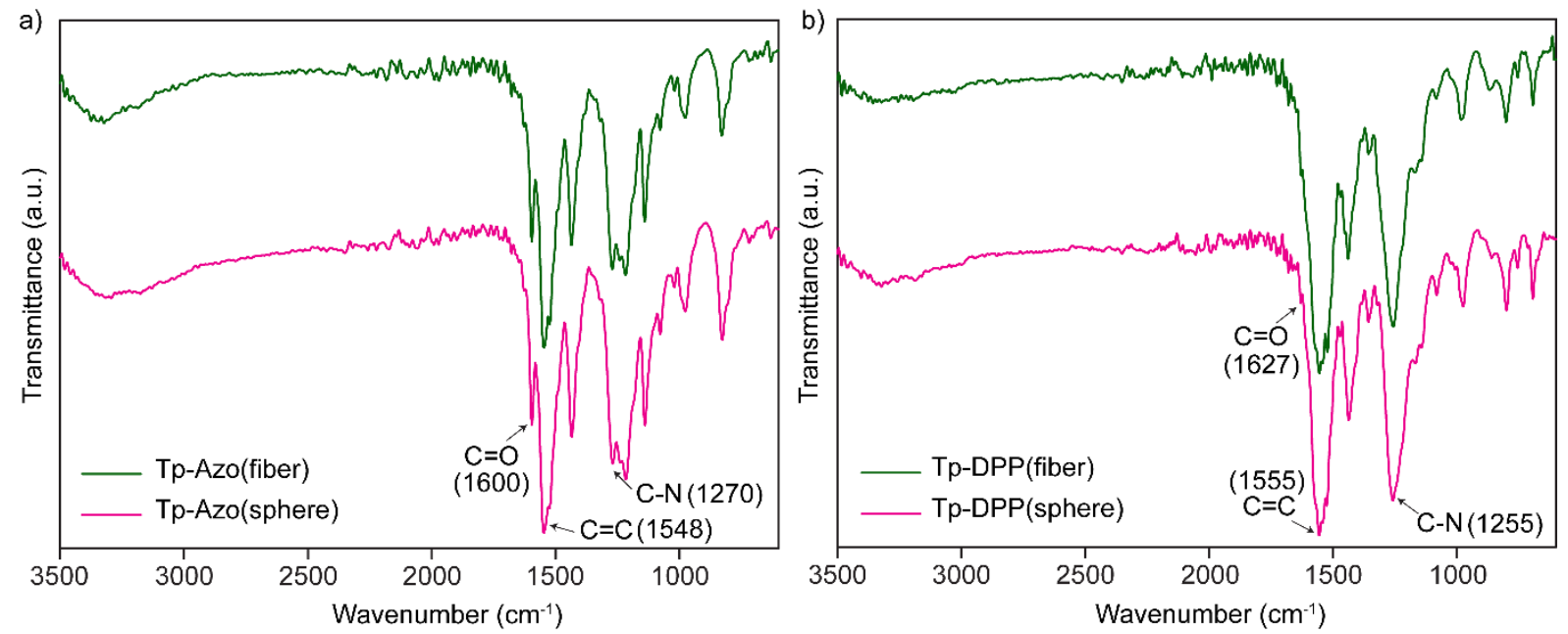

Figure S7 | FTIR spectra. FTIR spectra of the as-synthesized a) Tp-Azo and b) Tp-DPP COF thin-films show the characteristic peaks corresponding to $\beta$-ketoenamine-linked framework structures.

\section{Section S-5: TG analyses}
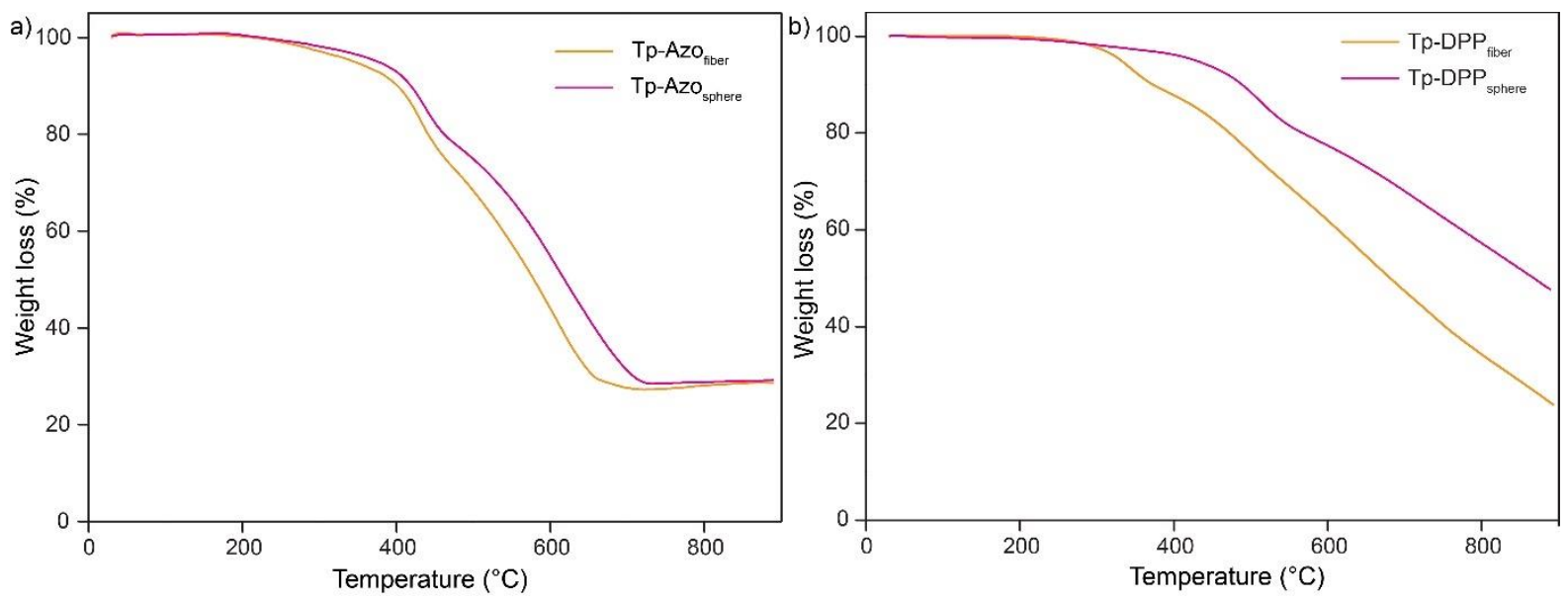

Figure S8 | TG analyses. TGA data of as-synthesized a) Tp-Azo b) Tp-DPP COF-films confirm the high thermal stability (minimum up to $\sim 300{ }^{\circ} \mathrm{C}$ ) under $\mathrm{N}_{2}$ atmosphere. Interestingly, $\mathrm{COF}_{\text {sphere }}$ films show higher thermal stability compared to the corresponding $\mathrm{COF}_{\text {fiber }}$ films. We attribute this to the combination of two factors i) larger crystallite growth both in plane as well as in the stacking direction (higher stacking energy) and ii) the higher covalent interconnection between individual nanospheres (high bond dissociation energy) leading to thermally robust $\mathrm{COF}_{\text {sphere }}$ films. 
Section S-6: Nitrogen adsorption analysis
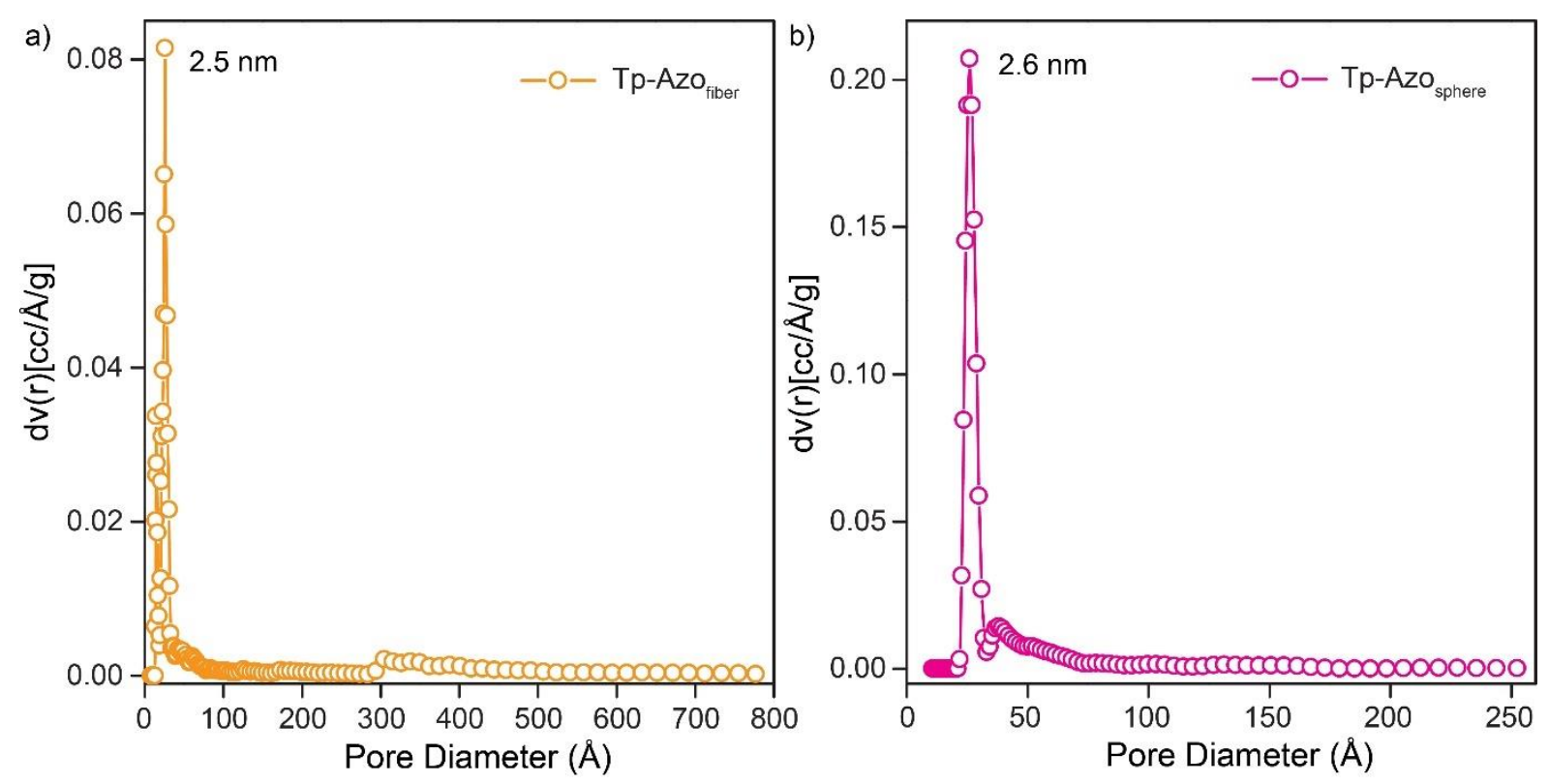

Figure S9 | Pore size distribution. Pore size distributions of a) Tp-Azofiber and b) Tp$\mathrm{AzO}_{\text {sphere }} \mathrm{COF}$ thin-films are calculated using the NLDFT model show a similar pore size distribution for films synthesized in both the methods. Tp-Azofiber films, the pore size shows a broader distribution of larger pores/voids (up to $\sim 80 \mathrm{~nm}$ ) as compared to the narrow

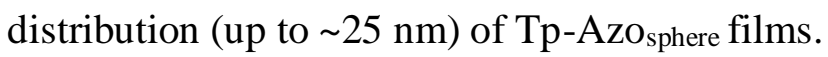
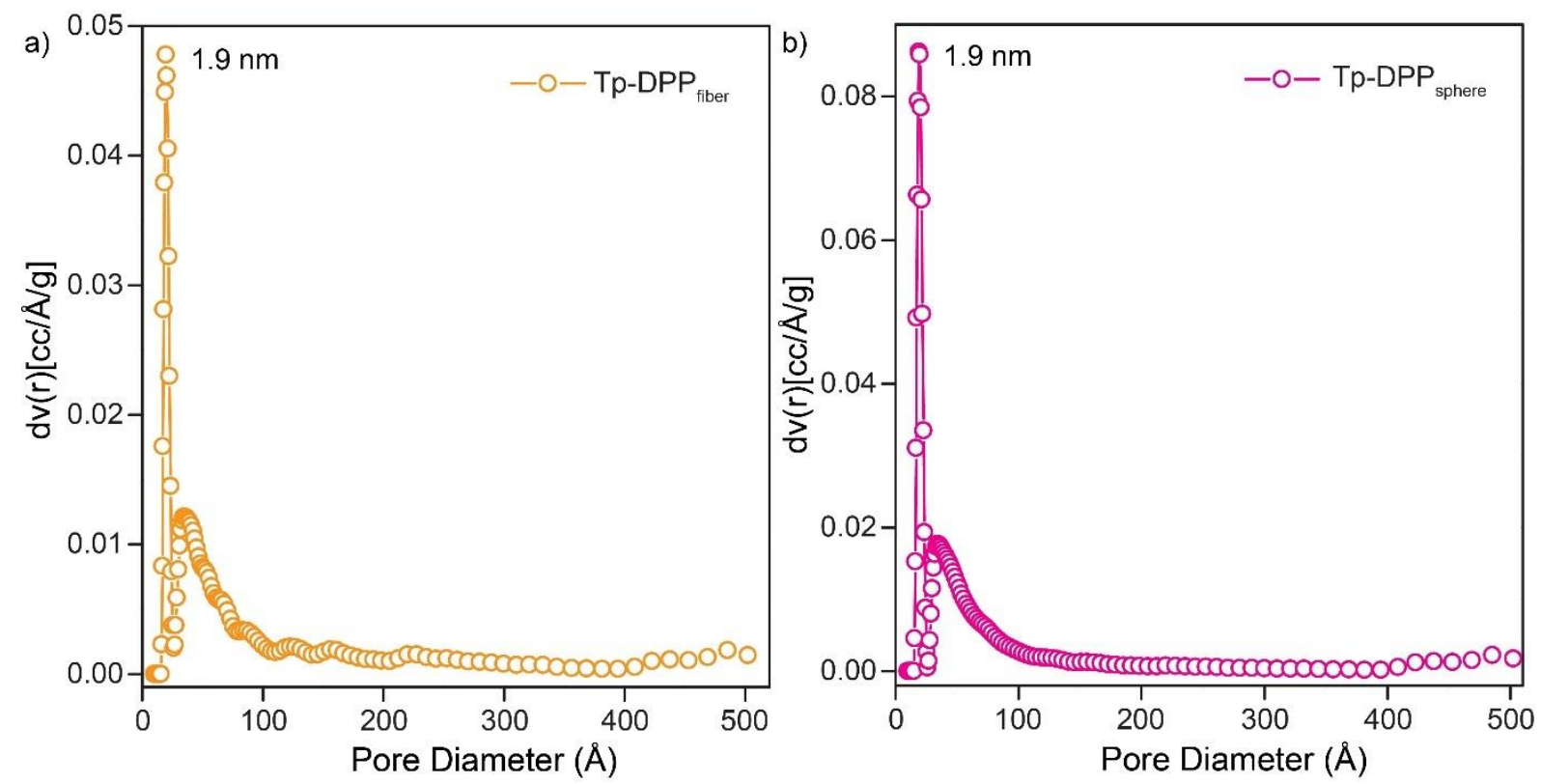

Figure S10 | Pore size distribution. Pore size distributions of a) Tp-DPP fiber and b) Tp$\mathrm{DPP}_{\text {sphere }} \mathrm{COF}$ thin-films are calculated using the NLDFT model show a similar pore size distribution for films synthesized in both the methods. The fraction of these larger 
pores/voids (e.g., between $\sim 3-15 \mathrm{~nm}$ ) present is higher $(\sim 25 \%)$ in Tp-DPP fiber as compared to the Tp-DPP ${ }_{\text {sphere }}(\sim 19 \%)$.

\section{Section S-7: SEM and TEM analyses}
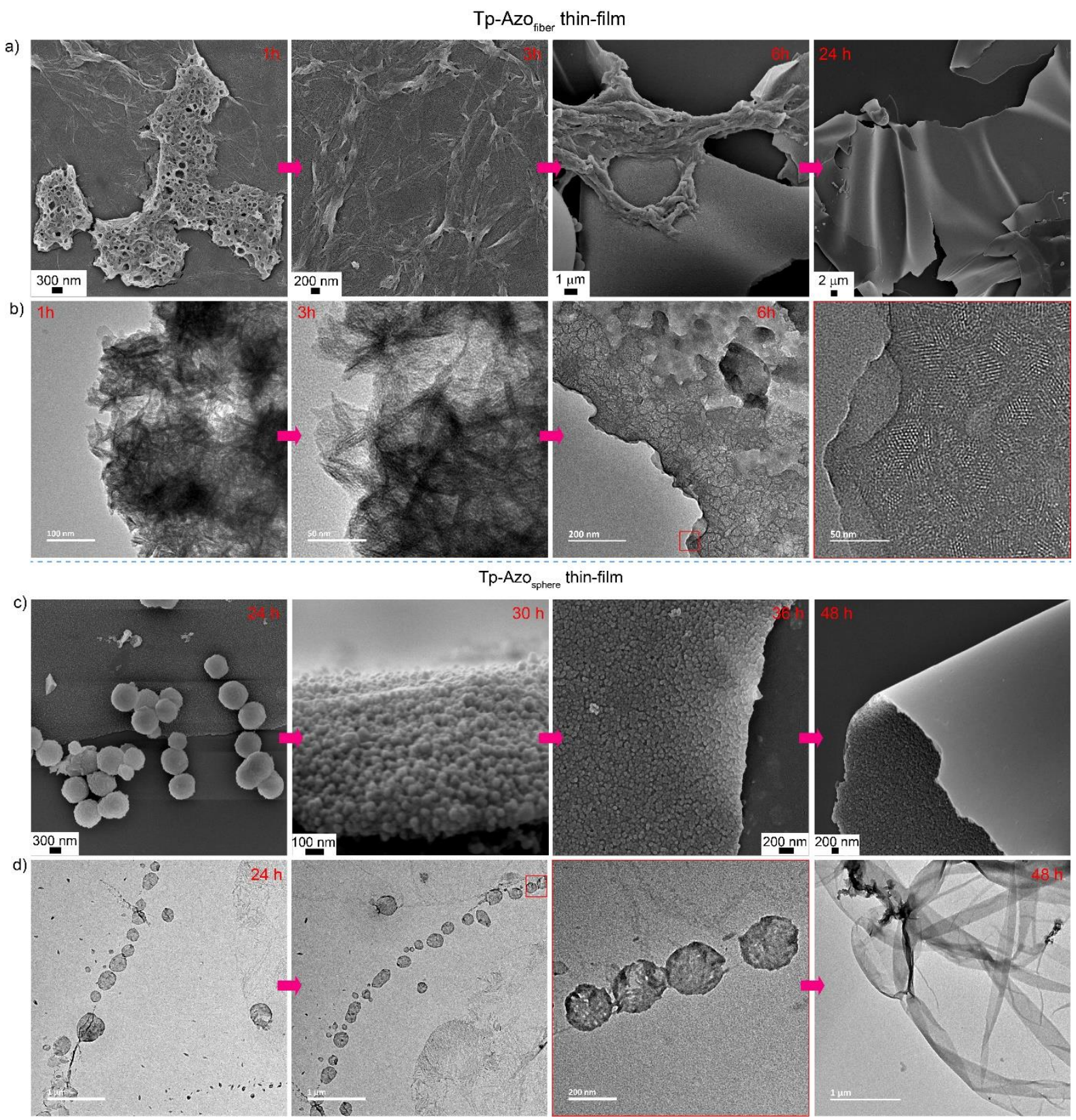

Figure S11 | Mechanism of Tp-Azo thin-film formation. a), b) SEM and TEM images of the Tp-Azofiber films (images taken with progress of time during the course of reaction). The images indicate a fiber based assembly to generate the thin-film. c), d) SEM and TEM images

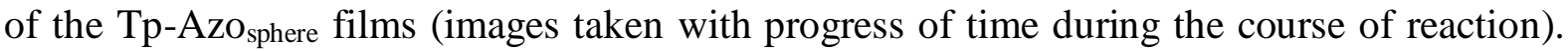
The images indicate a sphere-based assembly to generate the thin-film. 

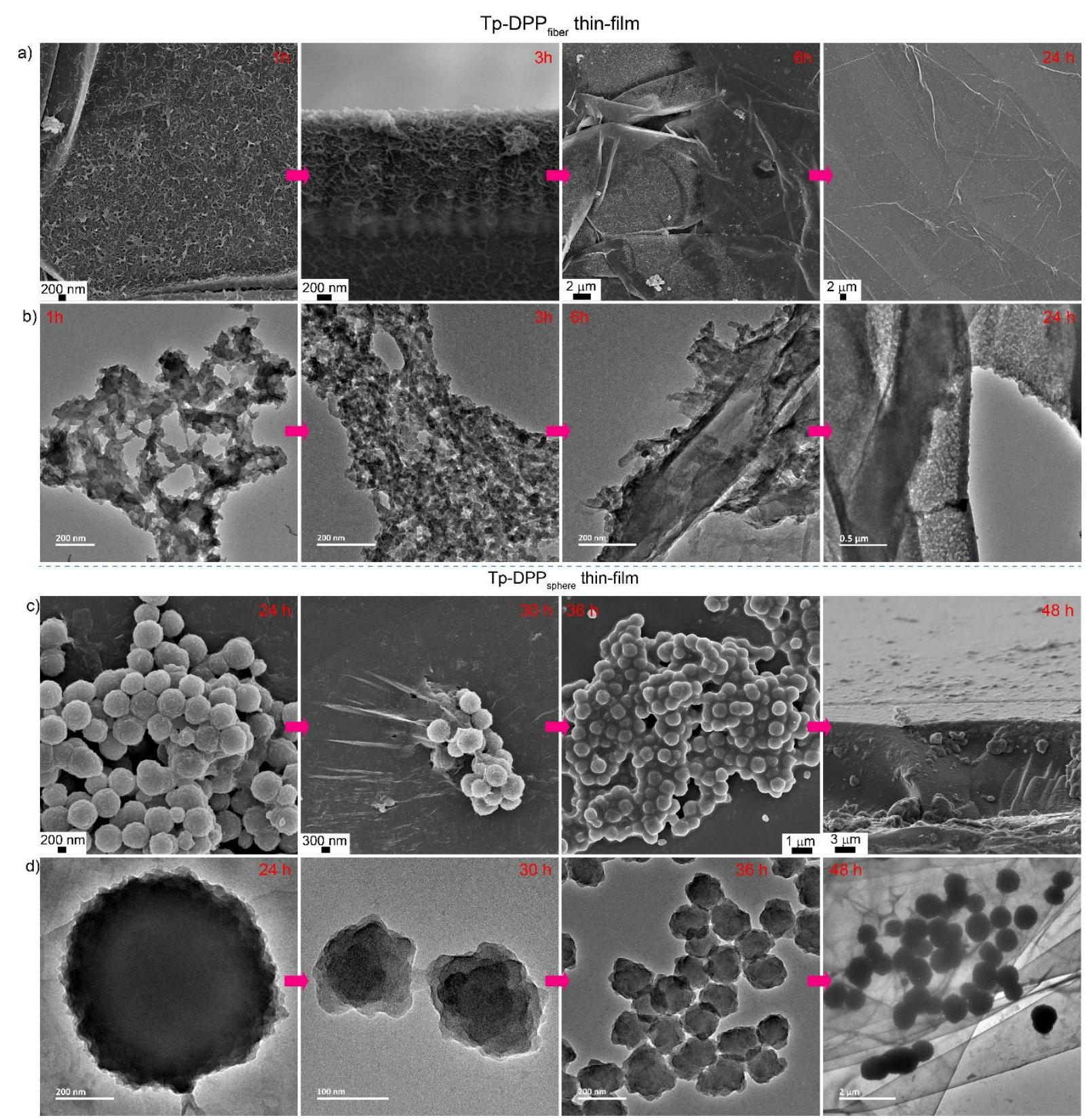

Figure S12 | Mechanism of Tp-DPP thin-films formation. a), b) SEM and TEM images of the Tp-DPP fiber films (images taken with progress of time during the course of reaction). The images indicate a fiber based assembly to generate the thin-film. c), d) SEM and TEM images of the Tp-DPP ${ }_{\text {sphere }}$ films (images taken with progress of time during the course of reaction). The images indicate a sphere-based assembly to generate the thin-film. 
Section S-8: AFM imaging
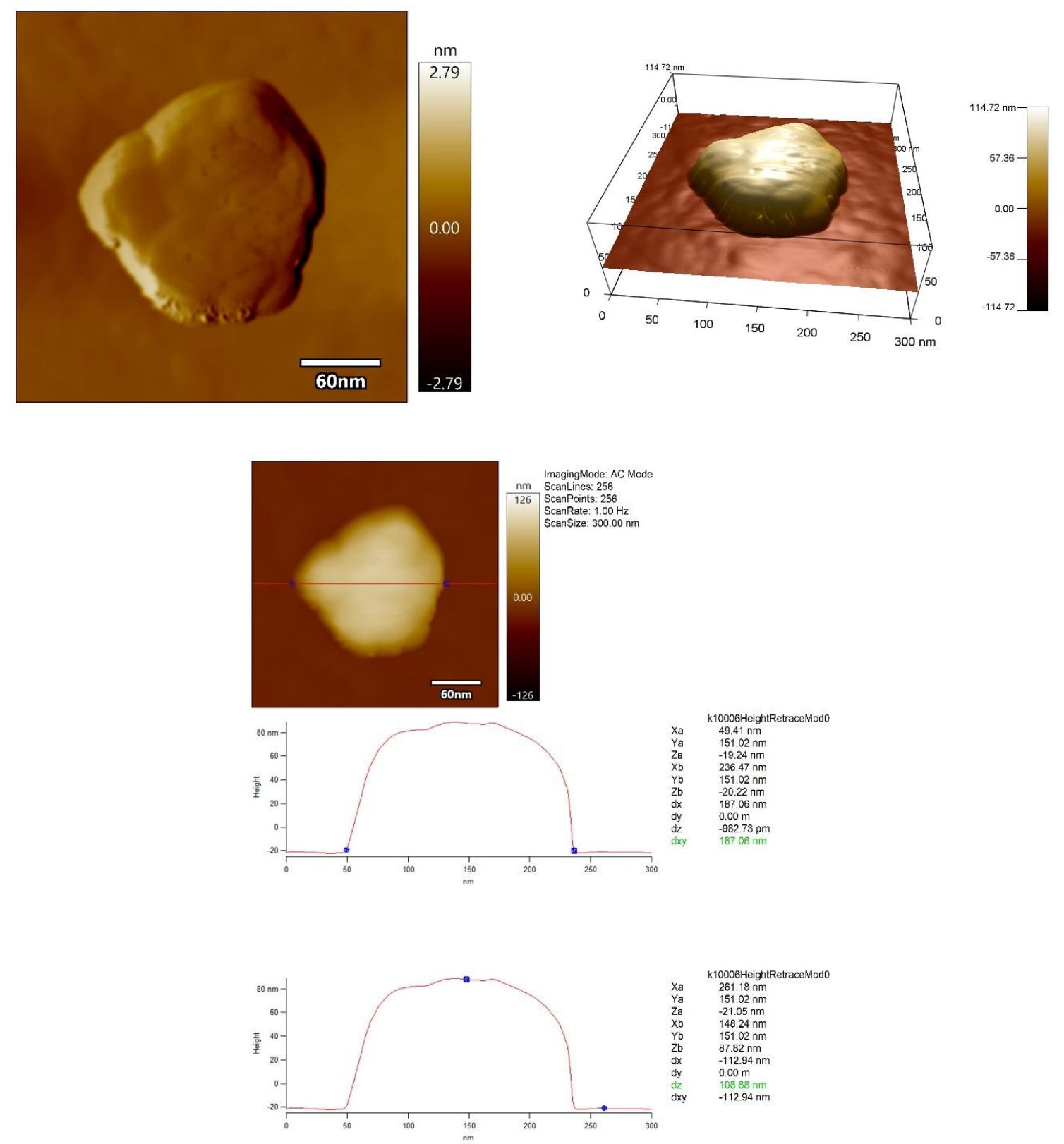

Figure S13 | AFM. Height image, 3D height profile image and amplitude image of the TpDPP nanospheres. 

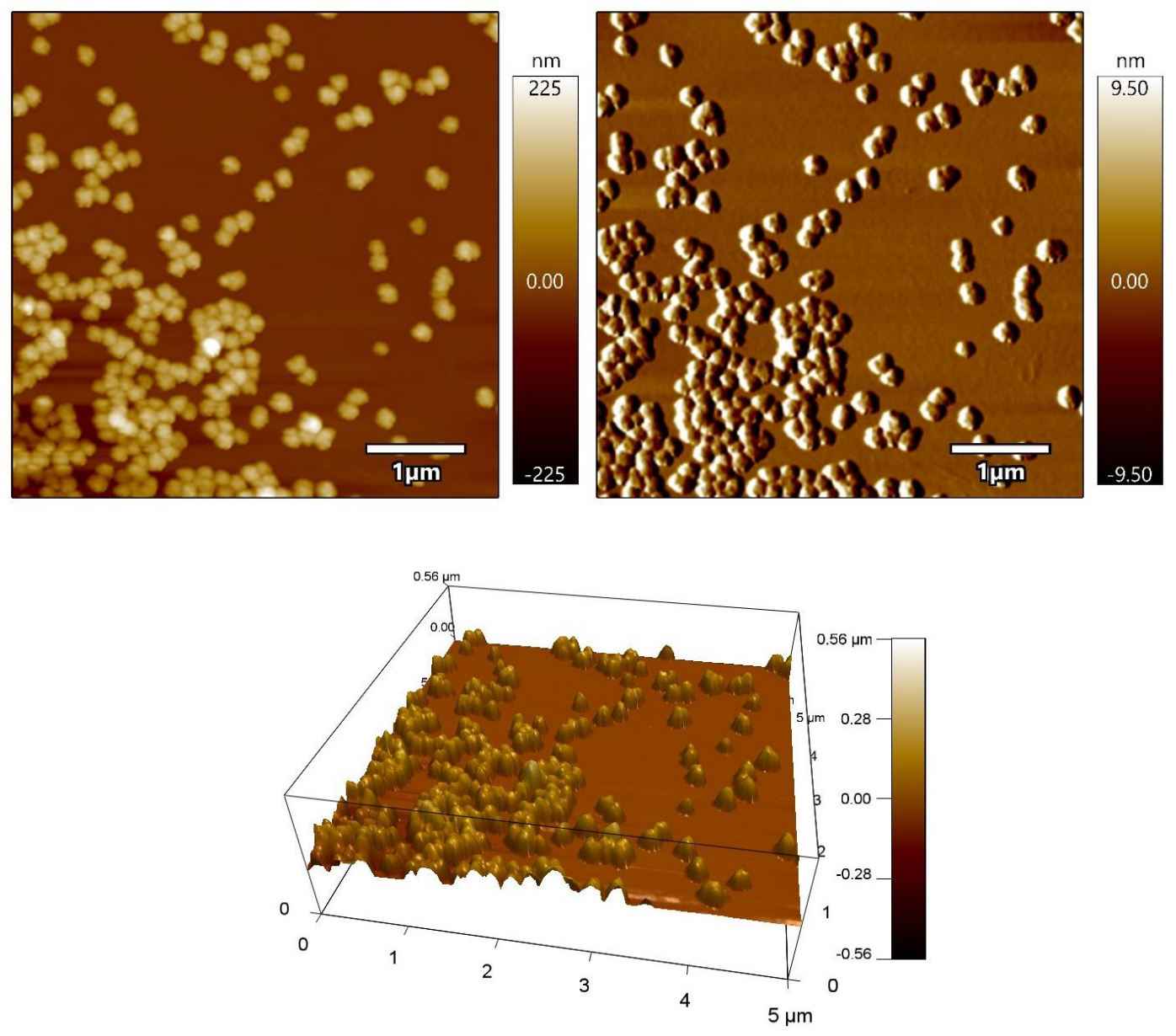

Figure S14 | AFM. Height image, amplitude image and 3D height profile image of the assembly of Tp-DPP nanospheres.
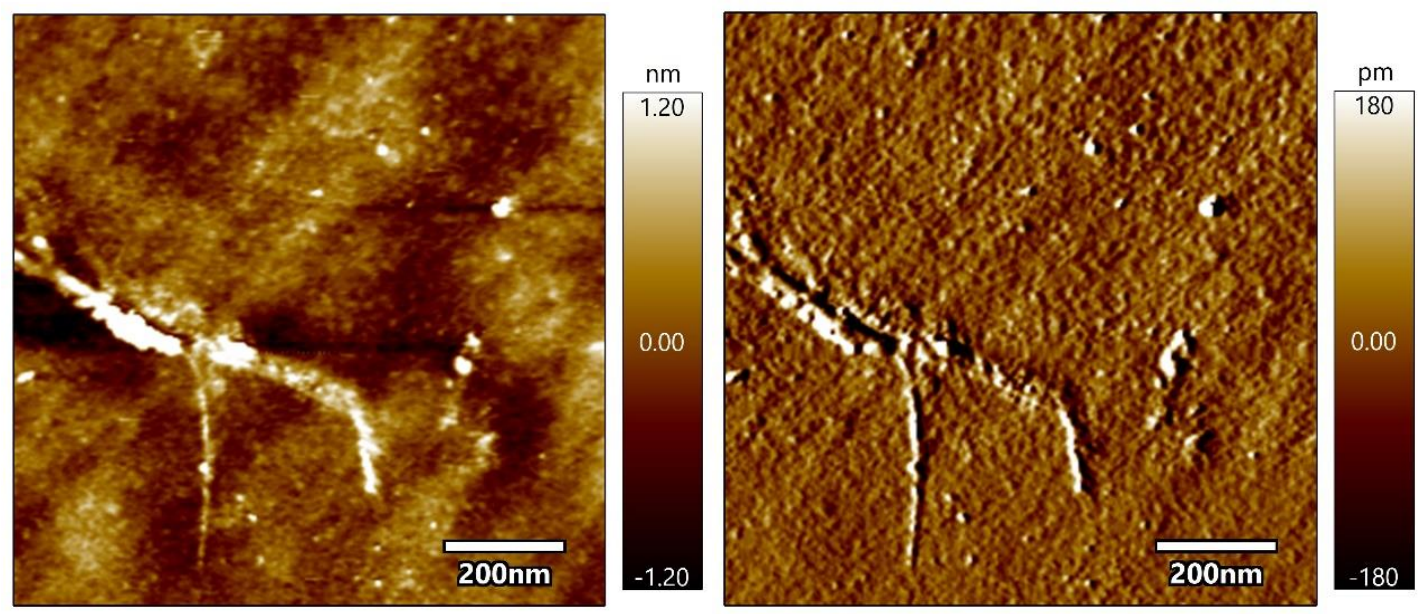

Figure S15 | AFM. Height image, amplitude image of the Tp-DPP nanofibers (collected during the progress of reaction before film formation). 
Section S-9: Dynamic Light Scattering data
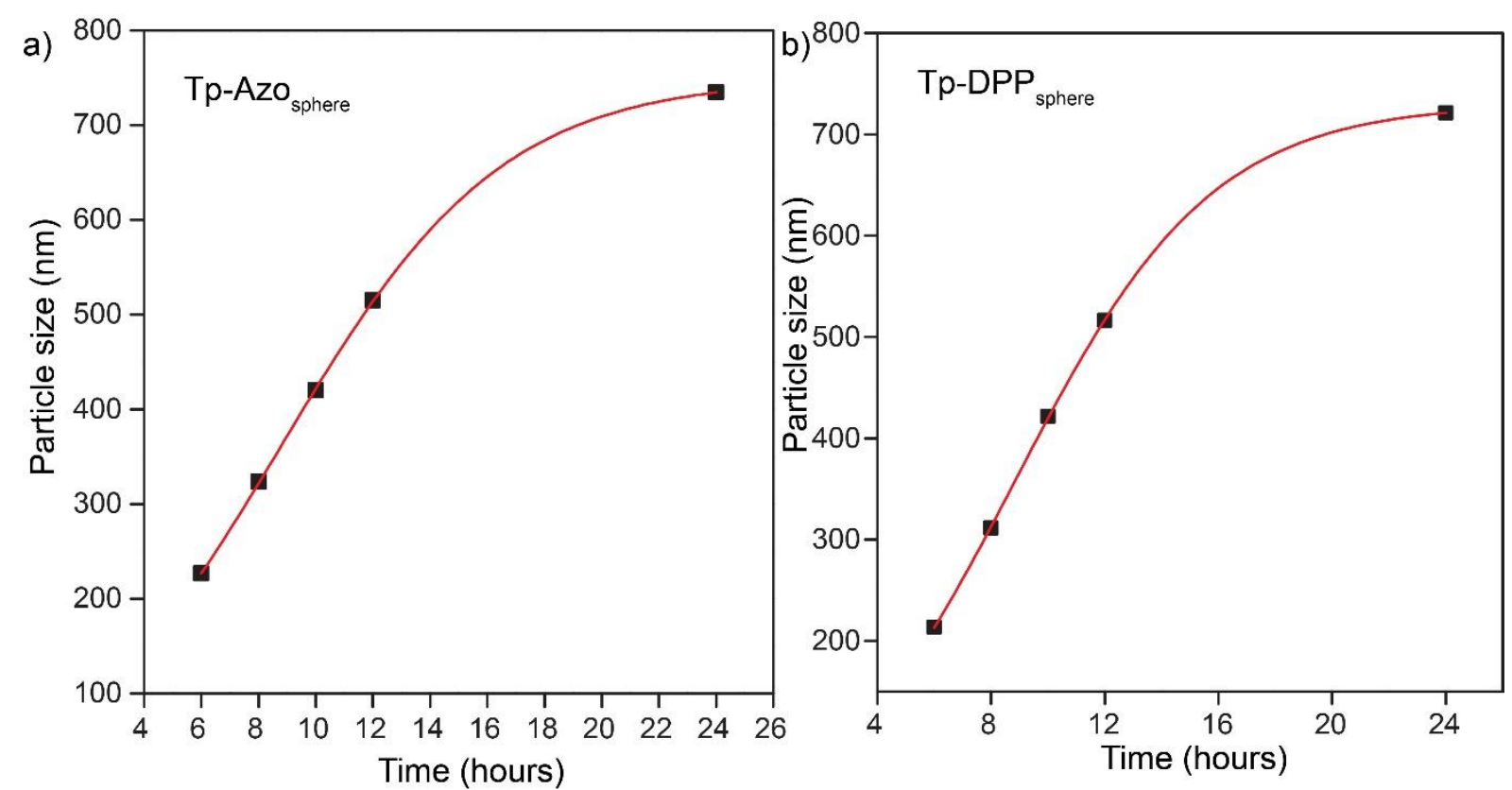

Figure S16 | DLS data of a) Tp-Azo and b) Tp-DPP COF nanospheres showing that with the progress of the reaction the size of the nanospheres increases and finally attain a stable size. 


\section{Section S-10: Nanoindentation experiments}

Nanoindentation $^{2,3}$ experiments have been done using the instrumentation set up from Hysitron Triboindentor, TI Premier, Minneapolis, USA equipped with an in situ Scanning Probe Microscope (SPM). The instrument measures the force and displacement of the nanoindentation probe with a unique three-plate capacitive transducer design which provides an unsurpassed noise floor $(0.2 \mathrm{~nm})$ and ultra-low working force $(\sim 75 \mathrm{nN})$. Three different tips were employed: a) Cono-spherical tip (tip radius $\sim 18 \mu \mathrm{m}$ ), b) Berkovich tip (tip radius $150 \mathrm{~nm}$ ) and another c) cono-spherical tip (tip radius $1 \mu \mathrm{m}$ ) for cross-validation of static indentation results. Analysis of the measured load vs. displacement $(P-h)$ curves (particularly the unloading segment) provides quantitative information of mechanical properties of the sample. The stiffness, $\mathrm{S}$, was calculated from the slope of the $P-h$ curve at the very initial point of unload (i.e. $\mathrm{S}=\mathrm{dP} / \mathrm{dh}$ ), therefore $E$ and $H$ can be found only at the maximum penetration depth. The elastic contact stiffness determined from the $P-h$ curves is later used to calculate the reduced modulus, $E_{r}$, by using the equation $E_{r}=(\sqrt{ } \pi / 2 \beta)(S / \sqrt{ } A)$, where, A, is the contact area under load (based on the calibrated tip area function) and $\beta$ is a constant that depends on the geometry of the indenter. The nanohardness, $H$, can be determined from the maximum indentation load, $P_{\max }$, by using the equation, $H=P_{\max } / A$, where the contact area (A) is a function of the contact depth, $h c$, and can be determined by the following equations, $\mathrm{A}=C_{0} h c^{2}+C_{1} h c+C_{2} h c^{1 / 2}+\cdots+C_{8} h c^{1 / 12}$, the constants will be varied with tip geometry. The contact depth, $h c$ can be determined by the equation $h c=h_{\max }-\varepsilon\left(P_{\max } / S\right)$ where, $h_{\max }$ is the maximum depth of penetration. Where $\varepsilon$ is a constant that depends on the geometry of the indenter. As the Poisson's ratio for the COF thin samples is unknown due to limited mechanical property studies currently, we have reported the machine generated reduced modulus or $E_{r}$ values as such in this manuscript. It is important to mention that the difference between reduced modulus and elastic modulus is small and the trends remain same. Hence the trends in reported reduced modulus values among different COFs will follow the trends of material's elastic modulus. Further, dynamic indentation (Nano DMA) was performed with $18 \mu \mathrm{m}$ cono-spherical tip to crosscheck the consistency of $E$ and $H$ data with respect to contact depth at different loads.

It is noteworthy to mention that for a blunt tip to extract hardness and reduced modulus value correctly deep indents are recommended. Generally, one should go one third of the tip radius. But in this case, due to the thickness restriction of the sample $(\sim 300 \mathrm{~nm})$, shallow indentation was performed. On the other hand, for very thin samples use of sharp tip 
is necessary to obtain precise information at shallow depth. But the softer nature of the films made the use of the sharper tips difficult in our study. We encountered two parallel challenges: one is the extreme softer behavior and another is the very low thickness of the samples. Nonetheless we could show the consistency of the load-depth curves obtained using the blunt conical tip across different kinds of thin films (Tp-Azo and Tp-DPP both). The absolute value of reduced modulus and hardness obtained using $18 \mu \mathrm{m}$ and $1 \mu \mathrm{m}$ conospherical tips might vary with the further increase of contact depth, however the load-depth response from the transducer with $0.2 \mathrm{~nm}$ resolution depicts relative mechanical behaviour of different films precisely.

Further evaluation by Berkovich tip (radius $150 \mathrm{~nm}$ ) on Tp-Azo films (spheres and fibres) confirmed the validity of the trends obtained using blunt cono-spherical tips. In this case the maximum depth of penetrations $(\sim 50-60 \mathrm{~nm})$ exceeded the one third of the tip radius $(\sim 45 \mathrm{~nm})$. After indentation on the surface, we could obtain the indent impressions by SPM imaging (a good impression reassures the reliability of the data). Therefore, the obtained reduced modulus and hardness values in our study are consistent and repeatable.

To avoid compliance issues with samples which typically arise due to poor surface adhesion of the sample on holder or tilting, the thin films were directly grown on glass slides and used directly for the indentation experiments.

For film like materials, the maximum depth of penetration is suggested to be $10-20 \%$ of the thickness of the film to ensure that the substrate contribution is absent or minimal. Since this is always a challenge for films, we carried out exhaustive tests varying the tip geometry (spherical and Berkovich) and test type (static indentation and dynamic indentation). However, since our samples are an order of magnitude softer as compared to the substrate, any substrate effect becomes obvious, and we did not see any alarming variations in our exhaustive study.

For all the films, we managed to fulfill the criteria by not allowing the tip to penetrate a depth of more than $\sim 10-20 \%$ of the thickness, but it was not possible in the case of Tp$\mathrm{DPP}_{\text {fiber }}$ films (thickness $280 \mathrm{~nm}$ ) due to its extreme mechanical softness. The required minimum load for restricting the tip penetration depth to $25-50 \mathrm{~nm}(\sim 10-20 \%)$ in this case is $\sim 2 \mu N$, which is instrument's setpoint value and too low to obtain any reliable data beyond noise level. Hence, we used a slightly higher load of $\sim 25 \mu \mathrm{N}$, which resulted in a depth of $\sim 200 \mathrm{~nm}$ (i.e., $90 \%$ ). 


\section{Experiment with blunt conospherical $(18 \mu \mathrm{m})$ tip}

\section{$\operatorname{Load}(p)-\operatorname{depth}(h)$ plot:}
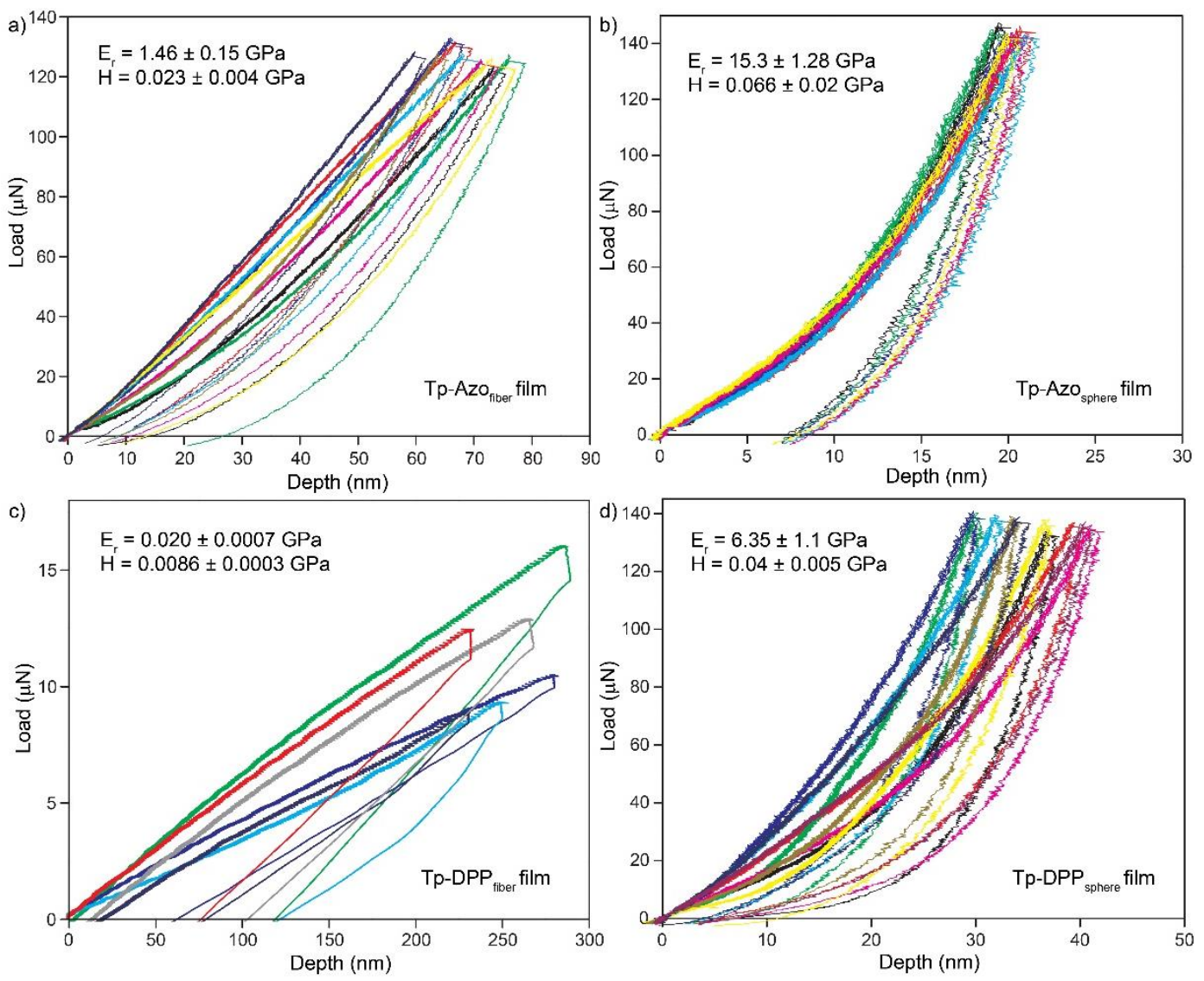

Figure S17 | $\operatorname{Load}(P)$-depth $(\boldsymbol{h})$ curve. Multiple load-depth curves performed on a) Tp-

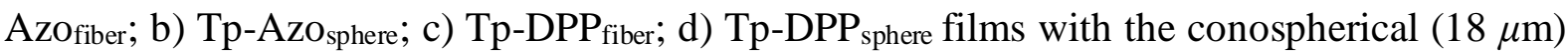
indenter tip. 


\section{Histogram plots:}

Tp-Azo sphere $_{\text {Film }}$

a)

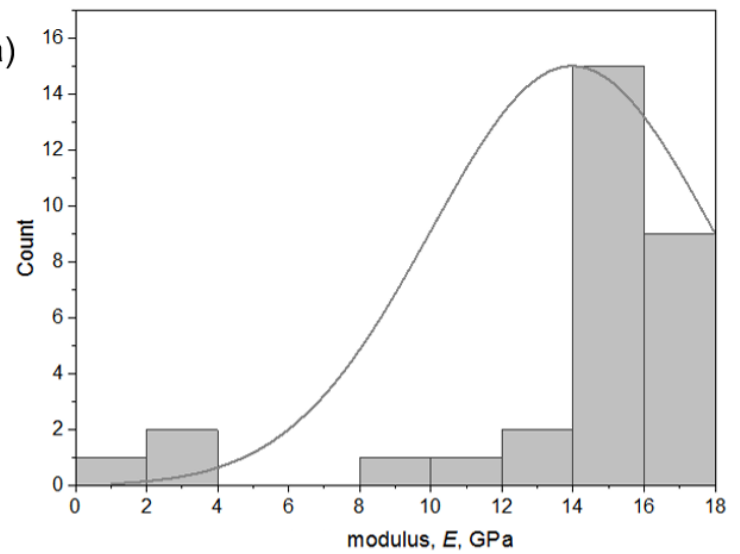

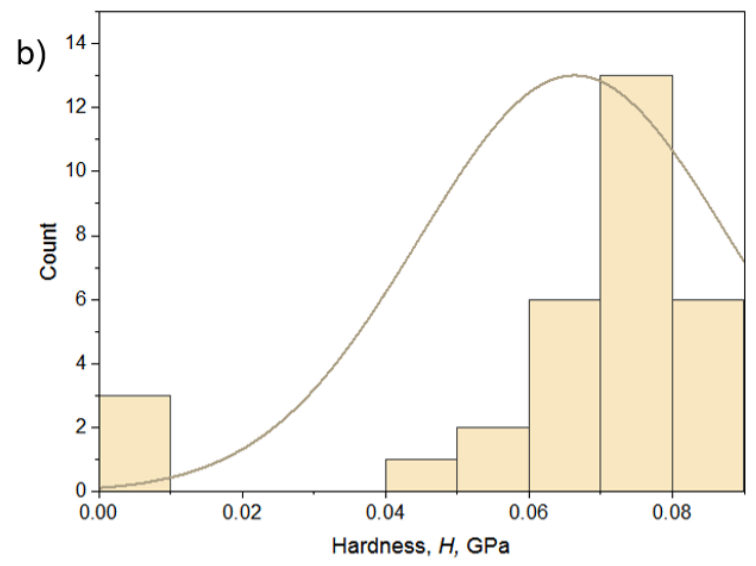

Tp-Azo ${ }_{\text {fiber }}$ Film

c)

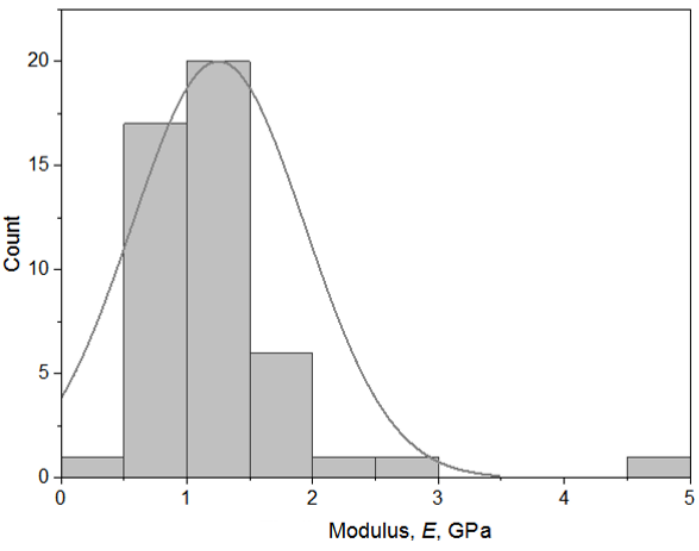

d)

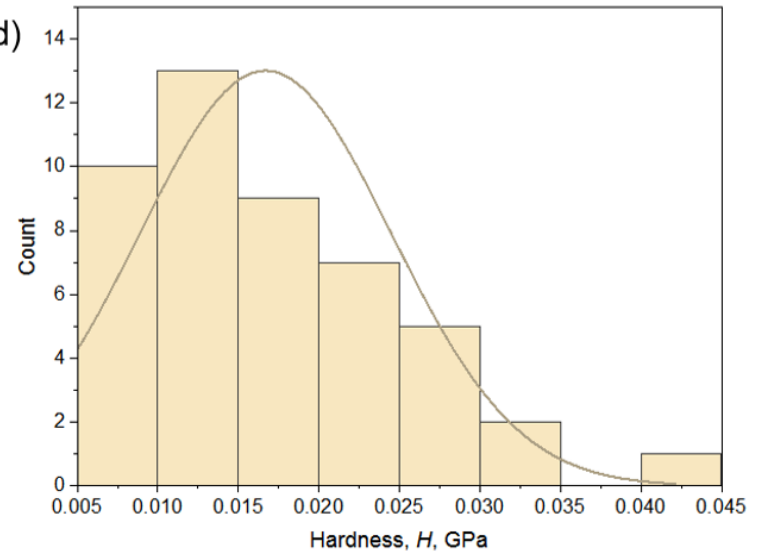

Figure S18 | Histogram plots of a), c) modulus and b), d) hardness values of multiple force curves in Tp-Azo thin films with the conospherical $(18 \mu \mathrm{m})$ indenter tip. 
Tp-DPP sphere $_{\text {Film }}$
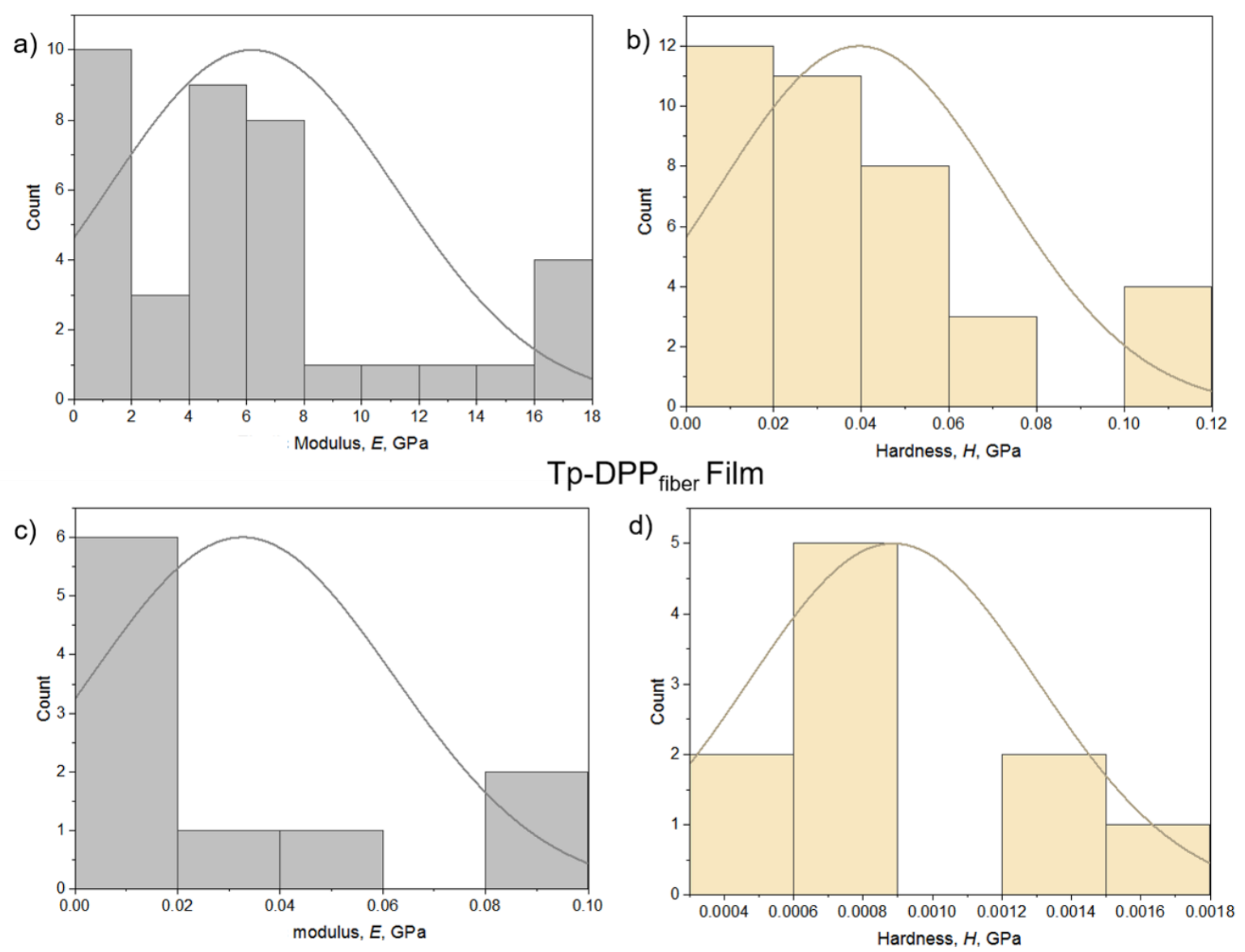

Figure S19 | Histogram plots of a), c) modulus and b), d) hardness values of multiple force curves in Tp-DPP thin films with the conospherical $(18 \mu \mathrm{m})$ indenter tip.
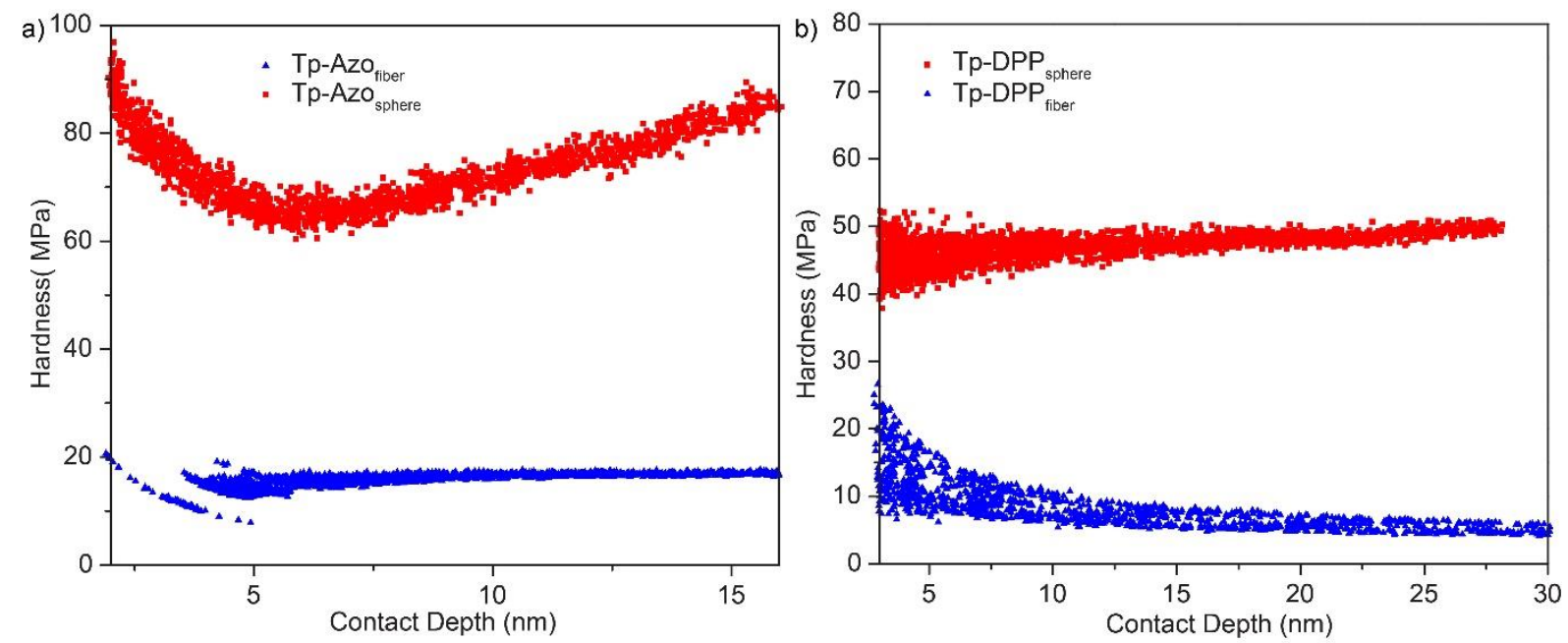

Figure S20 | Comparison of variation of hardness with contact depth in a) Tp-Azo; b) TpDPP thin-films with the conospherical $(18 \mu \mathrm{m})$ indenter tip. 


\section{Experiments with Berkovich $(150 \mathrm{~nm})$ tip}

\section{$\operatorname{Load}(p)-\operatorname{depth}(h)$ plot:}
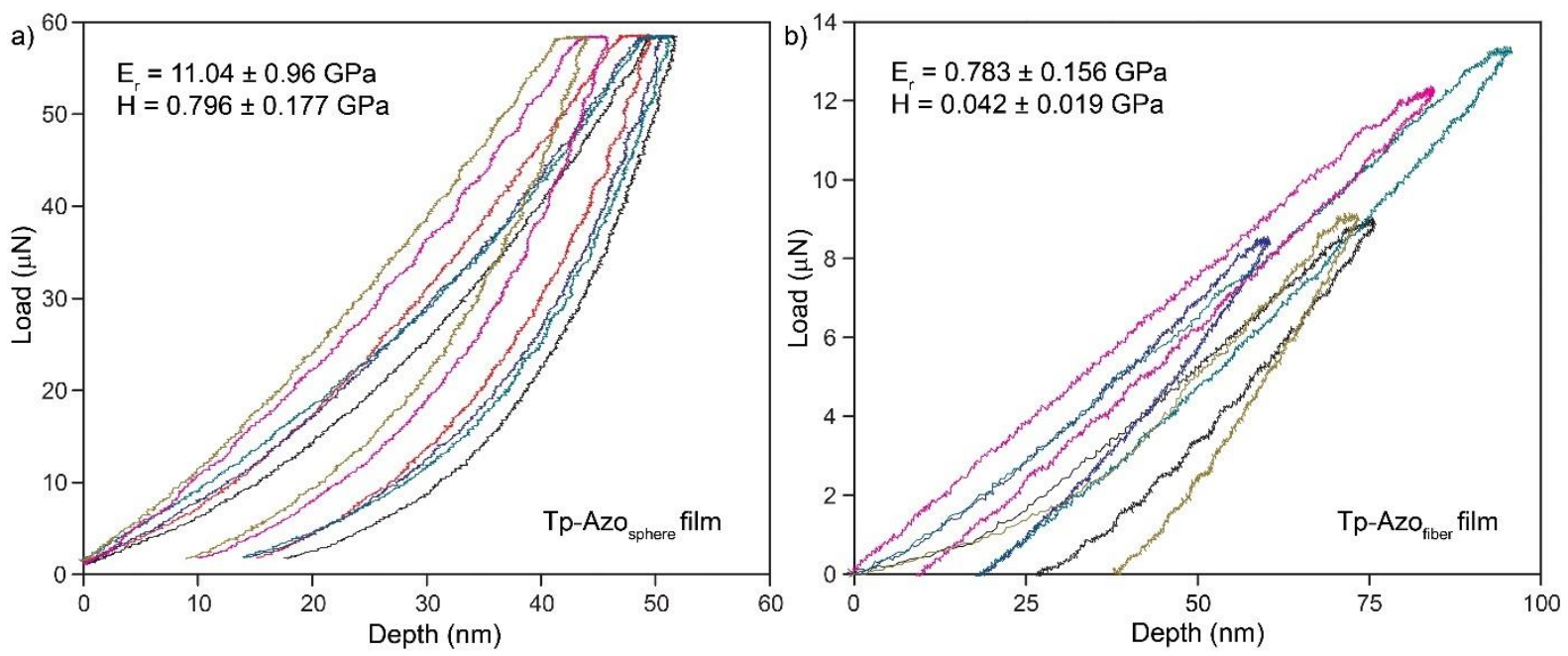

Figure S21 | $\operatorname{Load}(\boldsymbol{P})$-depth $(\boldsymbol{h})$ curve. Multiple load-depth curves performed on a) Tp$\mathrm{Azo}_{\text {sphere }}$; b) Tp-Azo fiber films with the Berkovich $(150 \mathrm{~nm})$ indenter tip.
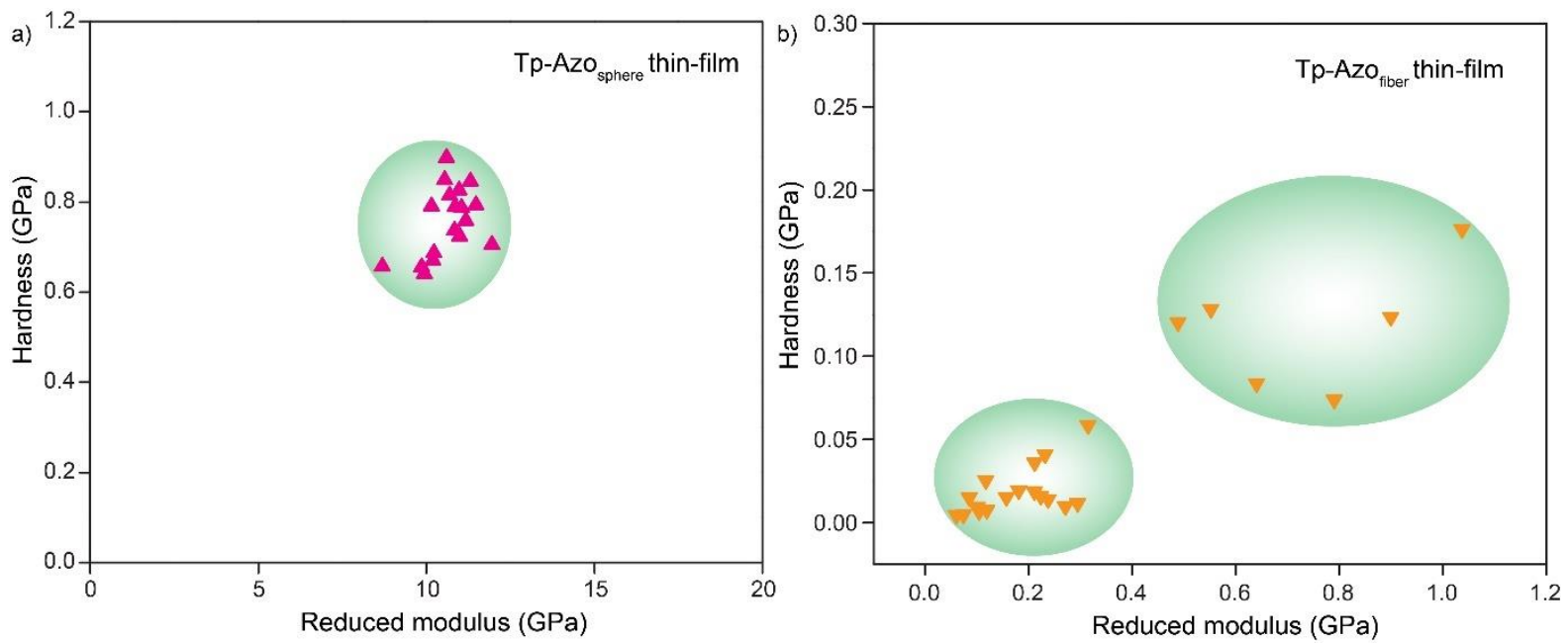

Figure S22 | Scattered data plots of hardness and modulus values derived from multiple loaddepth curves performed on a) Tp-Azo ${ }_{\text {sphere }}$ b) Tp-Azo fiber films with the Berkovich $(150 \mathrm{~nm})$ indenter tip. Tp-Azofiber films data indicate a more scattered data set consistent with the inhomogeneous nature of the fiber-film surface. 


\section{Histogram plots:}

Tp-Azo sphere $_{\text {Film }}$

a)

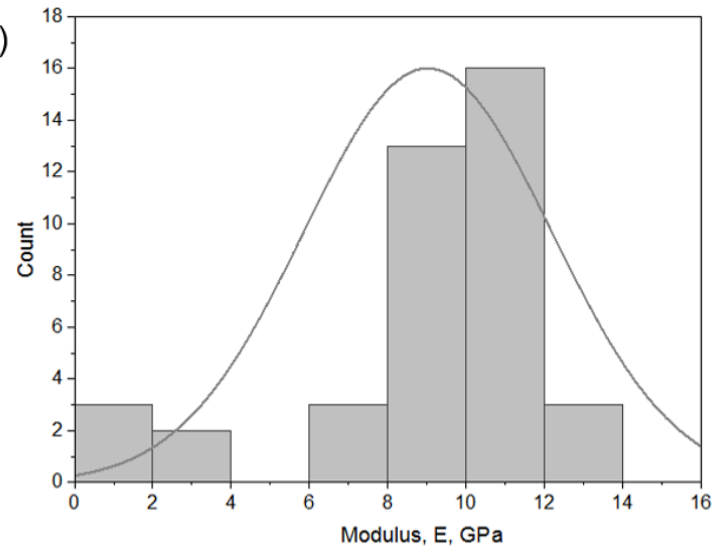

b)

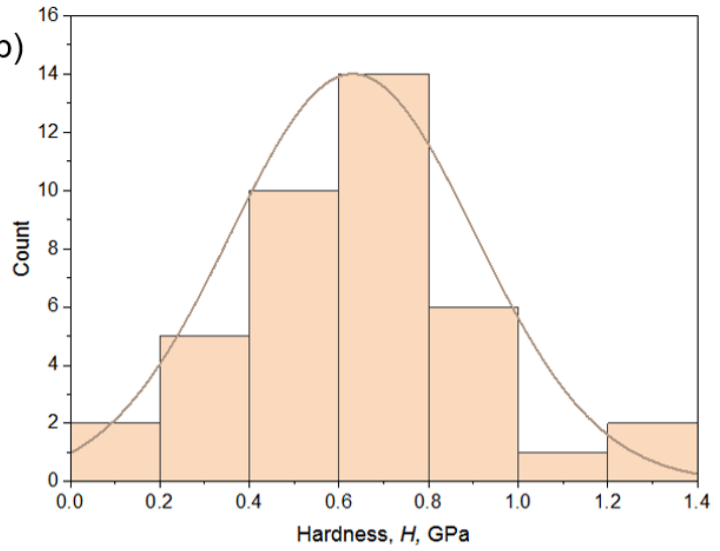

Tp-Azo fiber $_{\text {Film }}$

c)

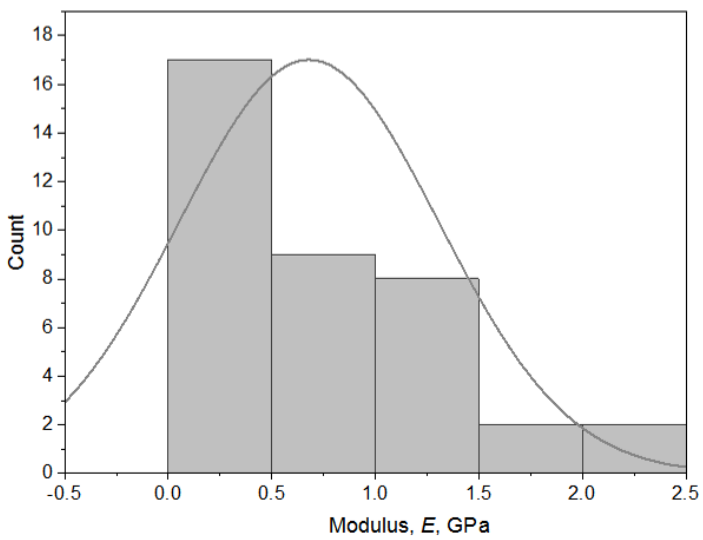

d)

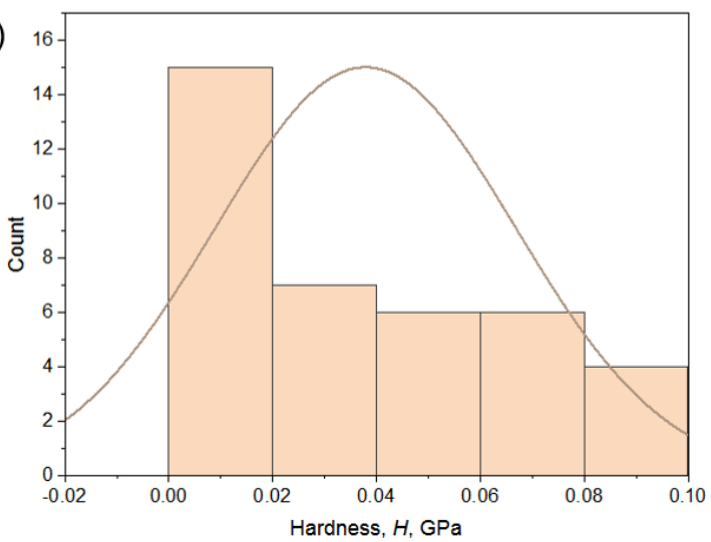

Figure S23 | Histogram plots of a), c) modulus and b), d) hardness values of multiple force curves in Tp-Azo thin films with the Berkovich indenter tip. 


\section{Experiments with conospherical $(1 \mu \mathrm{m})$ tip}

\section{$\operatorname{Load}(p)-\operatorname{depth}(h)$ plot:}
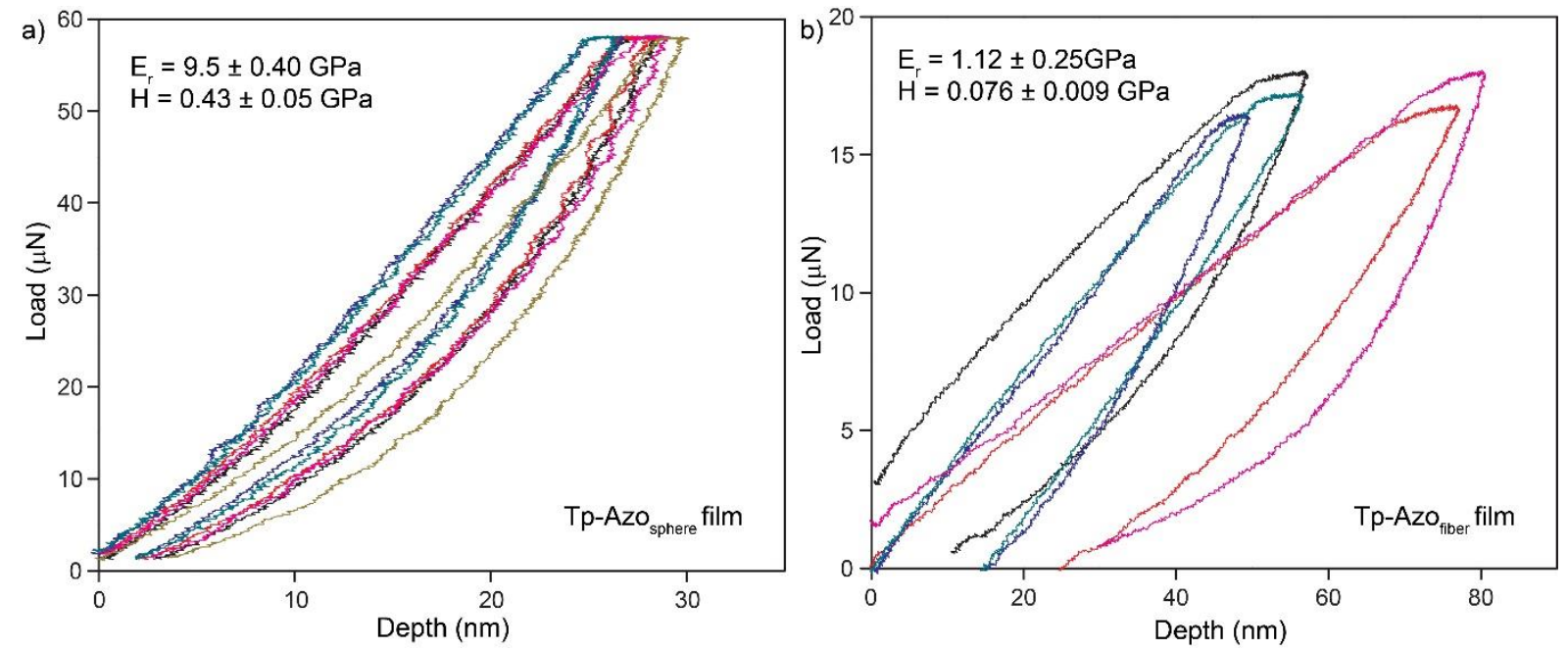

Figure S24 | $\operatorname{Load}(\boldsymbol{P})$-depth $(\boldsymbol{h})$ curve. Multiple load-depth curves performed on a) Tp-

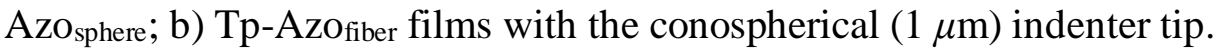
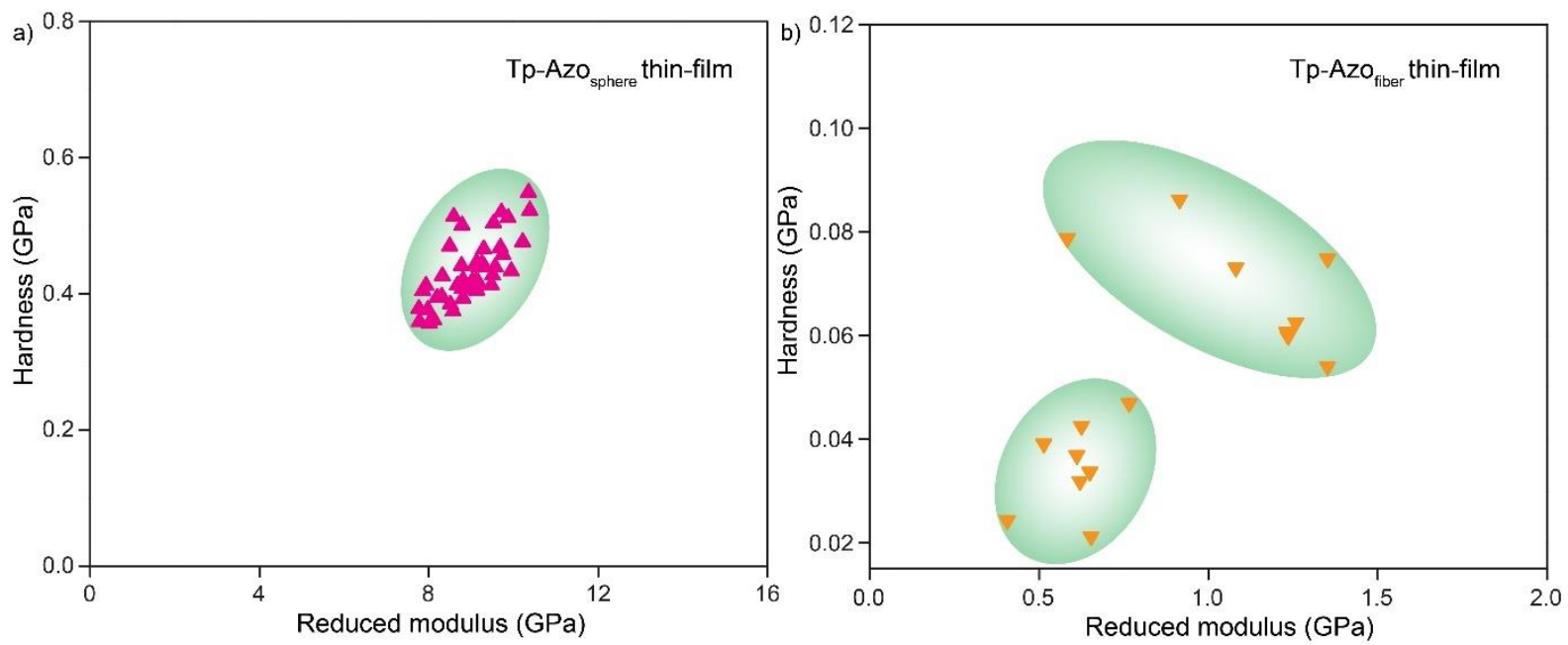

Figure S25 | Scattered data plots of hardness and modulus values derived from multiple load-

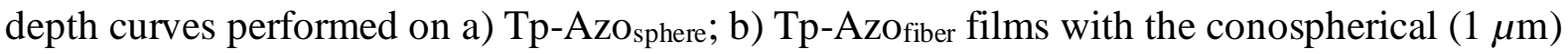
indenter tip. Tp-Azofiber films data indicate a more scattered data set consistent with the inhomogeneous nature of the fiber-film surface. 


\section{Histogram plots:}

Tp-Azo sphere $_{\text {Film }}$
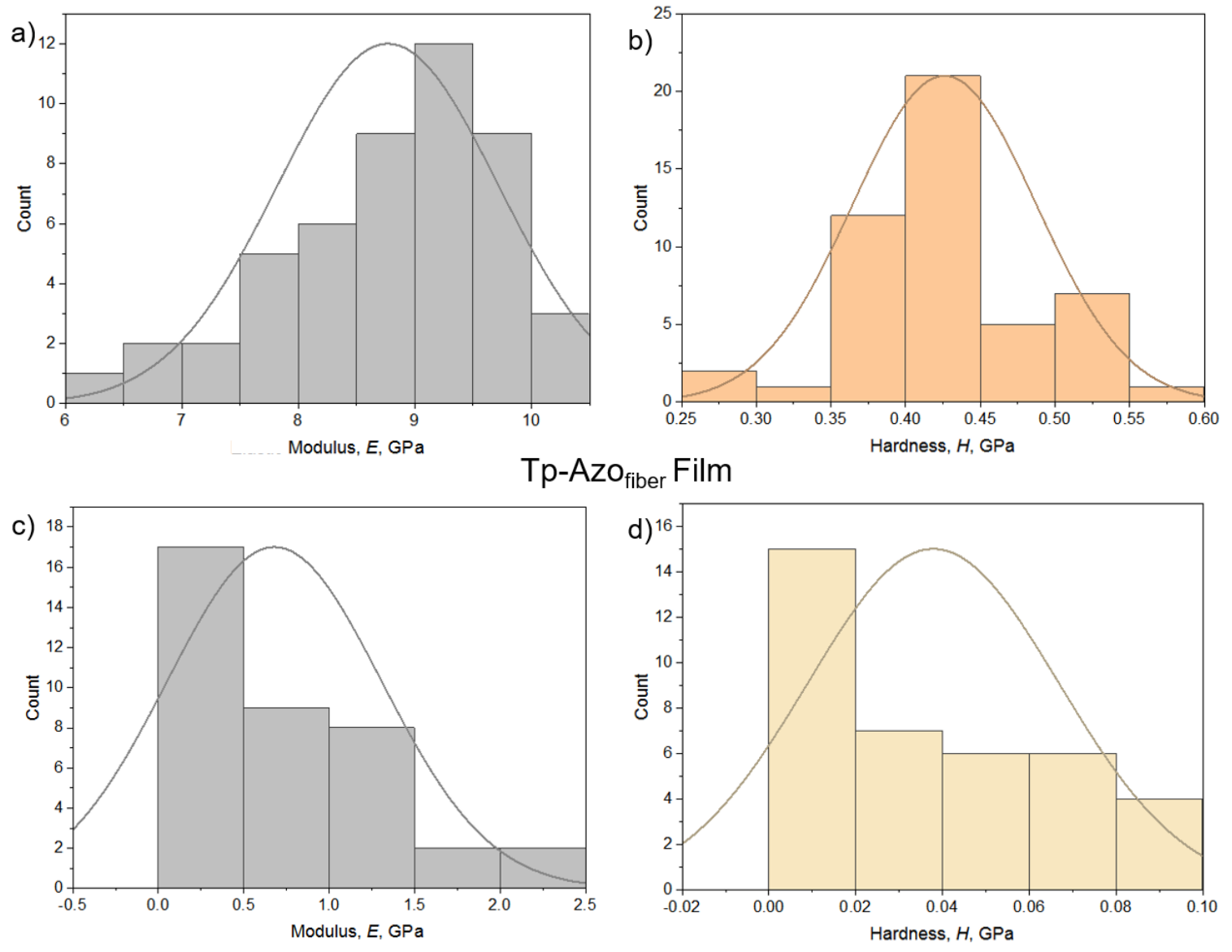

Figure S26 | Histogram plots of a), c) modulus and b), d) hardness values of multiple force curves in Tp-Azo thin films with the conopherical $(1 \mu \mathrm{m})$ indenter tip. 
Table-S1: Comparison of modulus $(E)$ and hardness $(H)$ values of different thin films.

\begin{tabular}{|c|c|c|c|c|c|c|}
\hline $\begin{array}{l}\text { COF thin- } \\
\text { film }\end{array}$ & $\begin{array}{c}\text { Probe } \\
\text { geometry and } \\
\text { radius }\end{array}$ & $\begin{array}{c}\text { Reduced } \\
\text { Modulus } \\
\text { (GPa) }\end{array}$ & $\begin{array}{c}\text { Hardness } \\
\text { (MPa) }\end{array}$ & $\begin{array}{l}\text { Thickness } \\
\text { of the } \\
\text { film (nm) }\end{array}$ & $\begin{array}{l}\text { Penetration } \\
\text { depth }(\mathrm{nm})\end{array}$ & $\begin{array}{c}\text { Percentage } \\
\text { of depth } \\
(\%)\end{array}$ \\
\hline \multirow{3}{*}{ Tp-Azo ${ }_{\text {sphere }}$} & $\begin{array}{l}\text { Cono-spherical } \\
\qquad(\sim 18 \mu \mathrm{m})\end{array}$ & $15.3 \pm 1.28$ & $66 \pm 2$ & $317 \pm 10$ & $25-40$ & $8-13$ \\
\hline & $\begin{array}{l}\text { Cono-spherical } \\
\qquad(\sim 1 \mu \mathrm{m})\end{array}$ & $9.5 \pm 0.40$ & $430 \pm 50$ & $317 \pm 10$ & $30-45$ & $10-15$ \\
\hline & $\begin{array}{l}\text { Berkovich } \\
(\sim 150 \mathrm{~nm})\end{array}$ & $11.0 \pm 0.96$ & $796 \pm 177$ & $317 \pm 10$ & $50-60$ & $16-19$ \\
\hline \multirow{3}{*}{ Tp-Azofibre } & $\begin{array}{l}\text { Cono-spherical } \\
\qquad(\sim 18 \mu \mathrm{m})\end{array}$ & $1.46 \pm 0.15$ & $23 \pm 4$ & $305 \pm 10$ & $65-70$ & $21-23$ \\
\hline & $\begin{array}{l}\text { Cono-spherical } \\
\qquad(\sim 1 \mu \mathrm{m})\end{array}$ & $1.12 \pm 0.25$ & $76 \pm 9$ & $305 \pm 10$ & $50-80$ & $16-26$ \\
\hline & $\begin{array}{l}\text { Berkovich } \\
(\sim 150 \mathrm{~nm})\end{array}$ & $0.78 \pm 0.15$ & $42 \pm 19$ & $305 \pm 10$ & $60-80$ & $20-26$ \\
\hline Tp-DPP ${ }_{\text {sphere }}$ & $\begin{array}{l}\text { Cono-spherical } \\
\qquad(\sim 18 \mu \mathrm{m})\end{array}$ & $6.35 \pm 1.1$ & $40 \pm 5$ & $230 \pm 10$ & $40-50$ & $17-22$ \\
\hline Tp-DPP fibre & $\begin{array}{l}\text { Cono-spherical } \\
\qquad(\sim 18 \mu \mathrm{m})\end{array}$ & $0.02 \pm 0.001$ & $8 \pm 0.3$ & $280 \pm 10$ & $220-250$ & $78-89$ \\
\hline
\end{tabular}


Table-S2: Comparison of modulus $(E)$ and hardness $(H)$ values with porous materials

\begin{tabular}{|c|c|c|c|c|c|}
\hline Material & $\begin{array}{c}\text { Specific } \\
\text { surface } \\
\text { area }\end{array}$ & $\begin{array}{l}\text { Modulus } \\
\text { (GPa) }\end{array}$ & $\begin{array}{c}\text { Hardness } \\
\text { (GPa) }\end{array}$ & Method & Reference \\
\hline $\begin{array}{c}\text { Poly }_{3 / 6} \mathrm{COF}- \\
42\end{array}$ & $479 \mathrm{~m}^{2} \mathrm{~g}^{-1}$ & 0.914 & - & Tensile & 4 \\
\hline $\begin{array}{c}\text { TTA-DHTA } \\
\text { COF }\end{array}$ & - & 25.9 & - & $\begin{array}{c}\text { AFM } \\
\text { Nanoindentation }\end{array}$ & 5 \\
\hline $\begin{array}{c}\text { TFPPy- } \\
\text { BBO-COF }\end{array}$ & $76 \mathrm{~m}^{2} \mathrm{~g}^{-1}$ & 10.6 & 1.15 & Nanoindentation & 6 \\
\hline $\begin{array}{c}\text { COF-505 } \\
\text { Demetalated- } \\
\text { COF-505 }\end{array}$ & - & $\begin{array}{c}12.5 \\
1.3\end{array}$ & - & $\begin{array}{c}\text { AFM } \\
\text { Nanoindentation }\end{array}$ & 7 \\
\hline ZIF-zni & $\begin{array}{c}\sim 1000- \\
1200 \mathrm{~m}^{2} \mathrm{~g}^{-1}\end{array}$ & $8-9$ & 1.1 & Nanoindentation & 8 \\
\hline $\begin{array}{l}\text { FPOP-100 } \\
\text { FPOP-101 }\end{array}$ & $\begin{array}{l}261 \mathrm{~m}^{2} \mathrm{~g}^{-1} \\
274 \mathrm{~m}^{2} \mathrm{~g}^{-1}\end{array}$ & $\begin{array}{l}3.4 \\
3.3\end{array}$ & $\begin{array}{l}0.23 \\
0.29\end{array}$ & Nanoindentation & 9 \\
\hline HKUST-1 & $\begin{array}{c}1500-1800 \\
\mathrm{~m}^{2} \mathrm{~g}^{-1}\end{array}$ & 9.3 & 0.46 & Nanoindentation & 10 \\
\hline QL4 fibers & - & 11.3 & 0.387 & Nanoindentation & 11 \\
\hline Collagen & - & $1.2-7$ & $0.03-3$ & $\begin{array}{c}\text { AFM } \\
\text { Nanoindentation }\end{array}$ & 12,13 \\
\hline $\begin{array}{l}\text { Amyloid } \\
\text { fibrils }\end{array}$ & - & $0.2-14$ & $0.1-1$ & $\begin{array}{c}\text { Bending/tensile } \\
\text { (AFM) }\end{array}$ & 14,15 \\
\hline $\begin{array}{c}\text { Tp-Azo sphere } \\
\text { thin-film }\end{array}$ & $1556 \mathrm{~m}^{2} \mathrm{~g}^{-1}$ & 15.3 & 0.066 & Nanoindentation & This work \\
\hline $\begin{array}{l}\text { Tp-DPP }{ }_{\text {sphere }} \\
\text { thin-film }\end{array}$ & $805 \mathrm{~m}^{2} \mathrm{~g}^{-1}$ & 6.35 & 0.040 & Nanoindentation & \\
\hline
\end{tabular}


Table-S3: Comparison of modulus $(E)$ and hardness $(H)$ values with polycrystalline thin-films

\begin{tabular}{|c|c|c|c|c|}
\hline $\begin{array}{c}\text { Polycrystalline } \\
\text { Thin-film }\end{array}$ & Modulus (GPa) & $\begin{array}{l}\text { Hardness } \\
\text { (GPa) }\end{array}$ & Method & Reference \\
\hline $\mathrm{Cu}(\mathrm{CHDA})$ & $10.9 \pm 3.1$ & $0.46 \pm 0.14$ & \multirow{3}{*}{ Nanoindentation } & \multirow{3}{*}{$\begin{array}{l}\text { J. Mater. Chem. } \\
\text { C, 2013, } 1,7716\end{array}$} \\
\hline $\mathrm{Cu}_{3}(\mathrm{BTC})_{2}$ & $3.5 \pm 2.5$ & $0.17 \pm 0.16$ & & \\
\hline $\mathrm{Cu}(\mathrm{INA})_{2}$ & $0.8 \pm 0.3$ & $0.02 \pm 0.01$ & & \\
\hline $\begin{array}{c}\text { MnDMS } \\
\text { nanosheets }\end{array}$ & $6-7$ & - & $\begin{array}{c}\text { AFM } \\
\text { nanoindentation }\end{array}$ & $\begin{array}{c}\text { ACS Nano } \mathbf{2 0 1 2}, \\
6,615\end{array}$ \\
\hline HKUST-1 & $9.6 \pm 1.1$ & $0.23 \pm 0.04$ & Nanoindentation & $\begin{array}{c}\text { Appl. Phys. Lett. } \\
\text { 2012, 101, } \\
101910\end{array}$ \\
\hline ZIF-8 & $3.44 \pm 1.42$ & - & \multirow[t]{2}{*}{ Nanoindentation } & \multirow{2}{*}{$\begin{array}{c}\text { Nat Commun } \\
\mathbf{2 0 1 9}, 10,3729\end{array}$} \\
\hline ZIF-67 & $3.79 \pm 1.83$ & - & & \\
\hline $\begin{array}{l}\text { Tp-Azo sphere } \\
\text { thin-film }\end{array}$ & 15.3 & 0.066 & Nanoindentation & \multirow{2}{*}{ This work } \\
\hline $\begin{array}{l}\mathrm{Tp}-\mathrm{DPP}_{\text {sphere }} \\
\text { thin-film }\end{array}$ & 6.35 & 0.040 & Nanoindentation & \\
\hline
\end{tabular}


Stress-strain response of the blank glass coverslip:

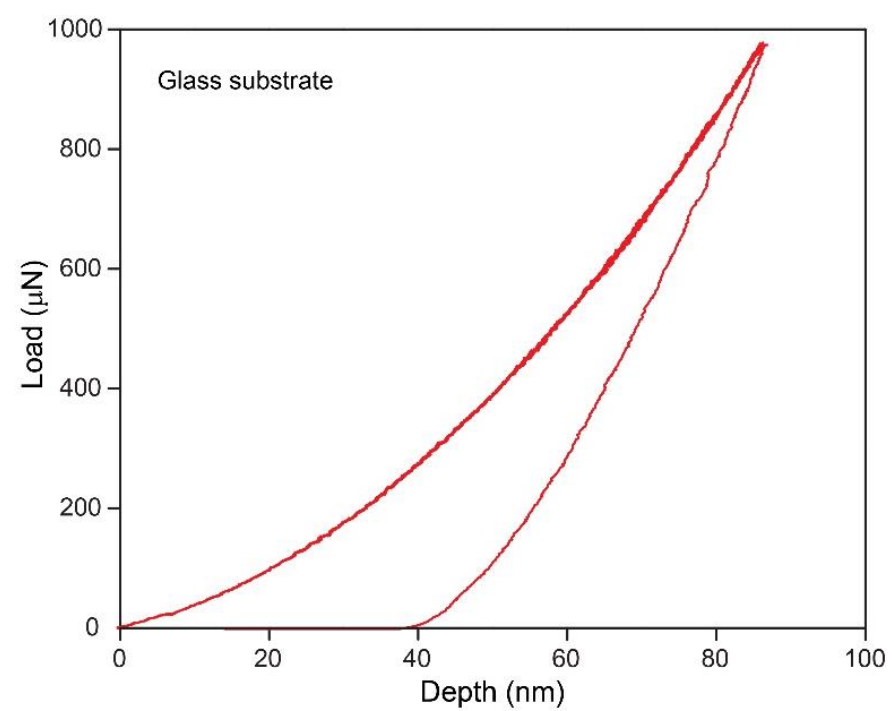

Figure S27 | Load-depth curve of blank glass substrate with Berkovich tip. The $E=65.6 \pm$ $1.1 \mathrm{GPa}$ and $H=6.0 \pm 0.27 \mathrm{GPa}$ indicate a much hard response as compared to the soft nature of the COF films. 


\section{Section S-11: Additional Data}
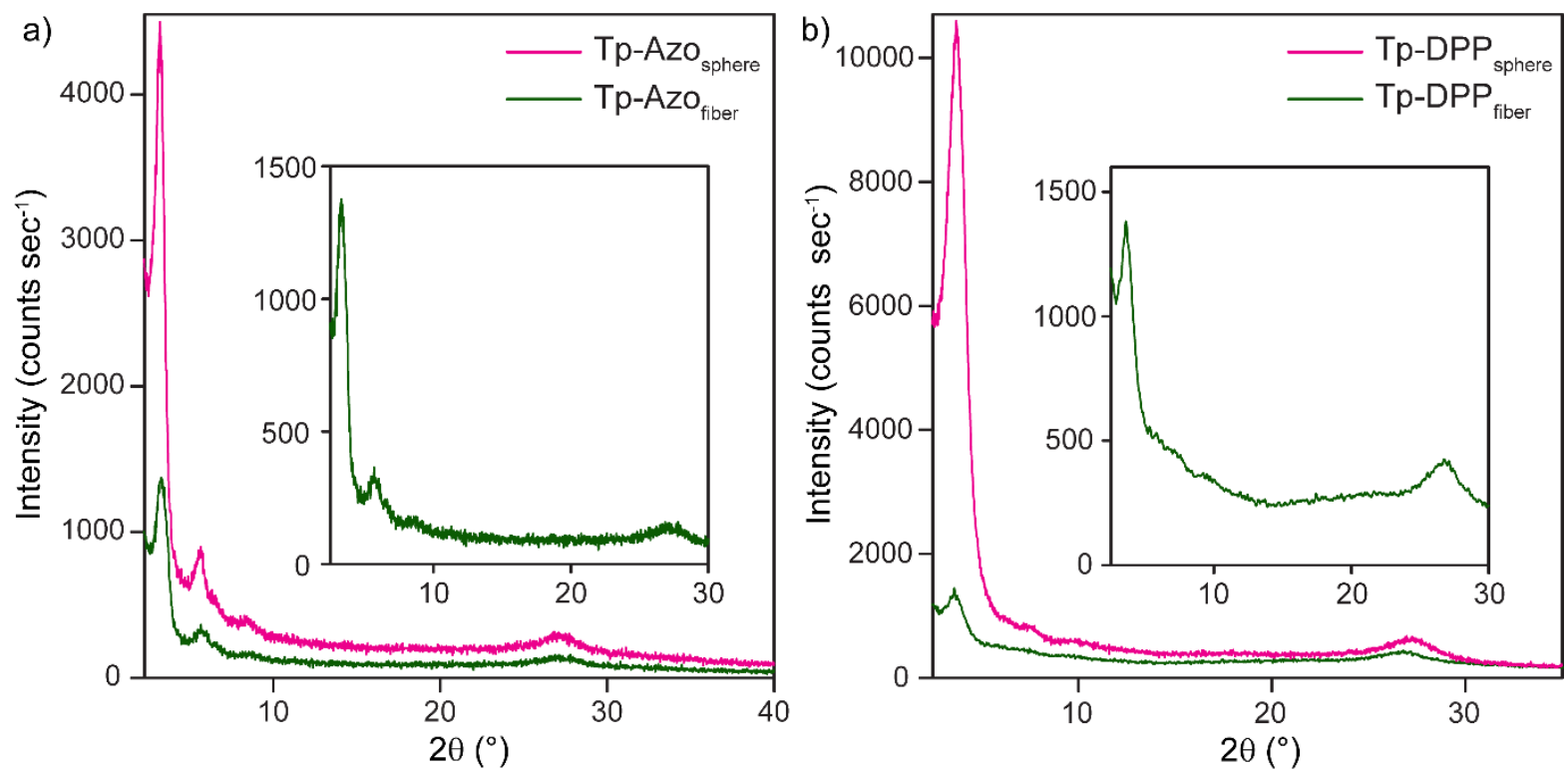

Figure S28: Comparison of PXRD plots between $\mathrm{COF}_{\text {fiber }}$ and $\mathrm{COF}_{\text {sphere }}$ thin films in a) TpAzo and b) Tp-DPP COFs, respectively.

We have compared the PXRD patterns of the $\mathrm{COF}_{\text {sphere }}$ and $\mathrm{COF}_{\text {fiber }}$ thin-films by plotting the absolute intensity of the diffraction peaks vs. the $2 \theta$ (Figure S28). As evident from the intensity differences, the $\mathrm{COF}_{\text {sphere }}$ films possess a much higher intensity count of the corresponding $<100>$ planes, which signifies the extended growth of the crystallites along the $\pi$ - $\pi$ stacking z-direction. 

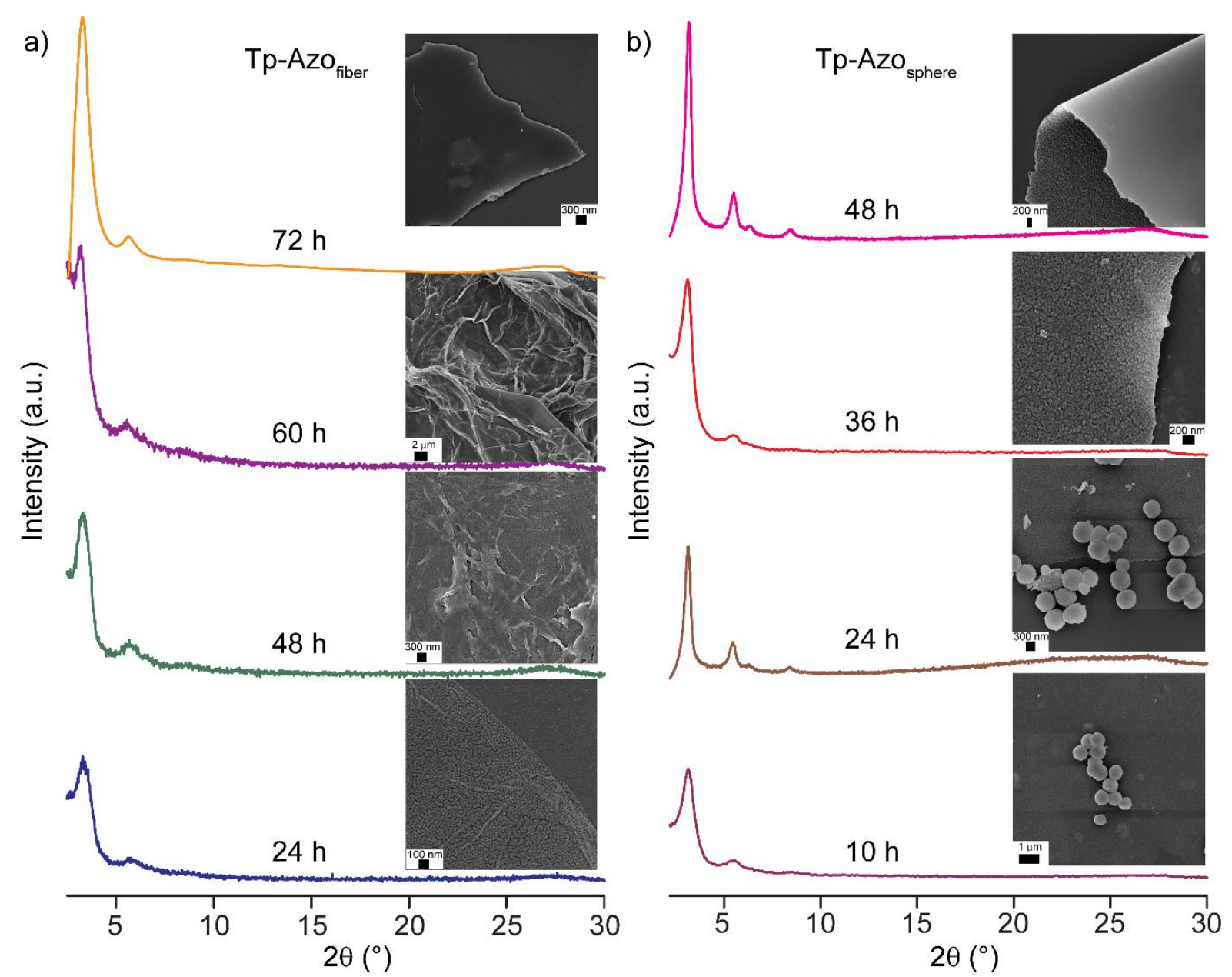

Figure S29: Time-dependent PXRD recorded at different intervals of time during the formation of a) Tp-Azo fiber and b) $\mathrm{Tp}-\mathrm{Azo}_{\text {sphere }}$ thin film.

Time-dependent PXRD in both fiber $\rightarrow$ film and sphere $\rightarrow$ film growth processes for the TpAzo COF (Figure S29) indicate that crystallinity originates at an earlier time scale during the sphere $\rightarrow$ film formation. The larger crystal growth is due to the higher temperature $(J . A m$. Chem. Soc. 2019, 141, 20371). The nucleation occurs rather slowly at room temperature for the fiber $\rightarrow$ film formation, and subsequently, the growth has also been delayed (J. Am. Chem. Soc. 2017, 139, 13083). 


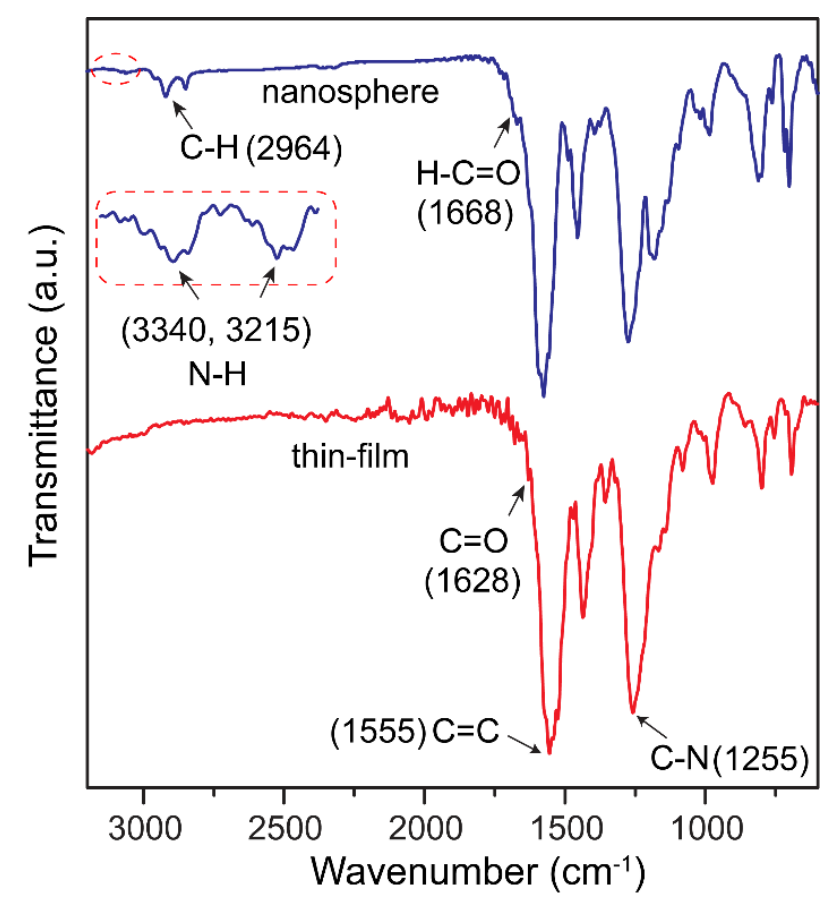

Figure S30: Comparison of FTIR spectra between nanosphere and thin films in Tp-DPP COF showing the disappearance of $-\mathrm{N}-\mathrm{H}$ and $\mathrm{H}-\mathrm{C}=\mathrm{O}$ peaks, indicating $=\mathrm{C}-\mathrm{N}$ bond formation during the sphere $\rightarrow$ film assembly process.

Comparison of the FTIR spectra (Figure S30) between the nanosphere and thin-film indicate the absence of free $-\mathrm{N}-\mathrm{H}\left(3340,3215 \mathrm{~cm}^{-1}\right),-\mathrm{C}-\mathrm{H}\left(2964 \mathrm{~cm}^{-1}\right)$, and $\mathrm{H}-\mathrm{C}=\mathrm{O}\left(1669 \mathrm{~cm}^{-1}\right)$ groups in the thin-films (J. Am. Chem. Soc. 2019, 141, 20371). The thin-films possess a shifted $\mathrm{C}=\mathrm{O}$ stretching frequency at $1628 \mathrm{~cm}^{-1}$, suggesting that the free $-\mathrm{N}-\mathrm{H}$ and $\mathrm{H}-\mathrm{C}=\mathrm{O}$ in the nanosphere surface undergo covalent bond formation to generate the thin film. 

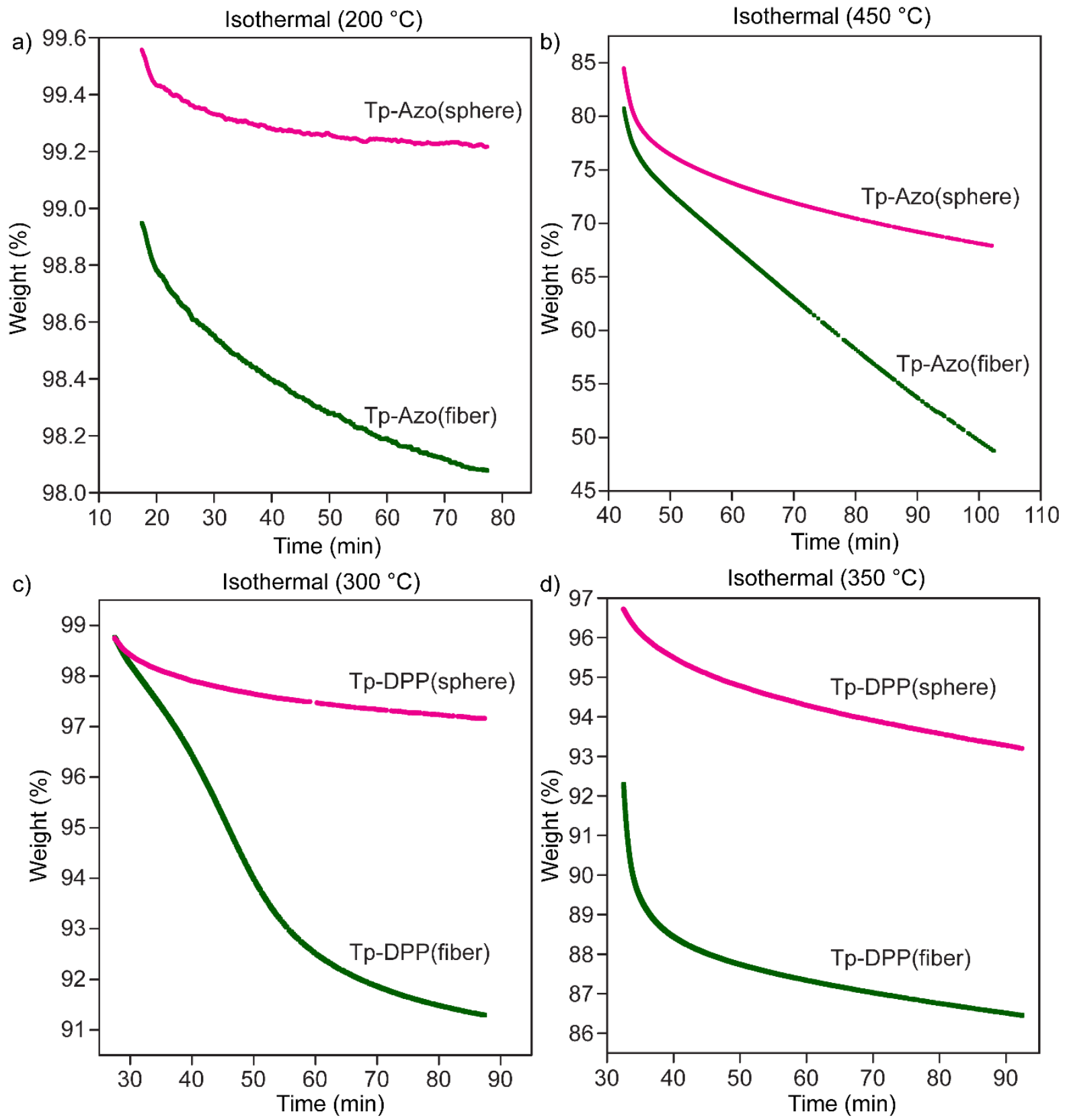

Figure S31: Comparison of isothermal degradation between $\mathrm{Tp}-\mathrm{AzO}$ fiber and $\mathrm{Tp}-\mathrm{AzO}_{\text {sphere }}$ thin films at a) $200{ }^{\circ} \mathrm{C}$ and b) $450{ }^{\circ} \mathrm{C}$; Tp-DPP fiber and Tp-DPP sphere thin films at c) $300{ }^{\circ} \mathrm{C}$ and d) $350{ }^{\circ} \mathrm{C}$ indicating a faster degradation rate for the $\mathrm{COF}_{\text {fiber }}$ films.

By comparing the mass loss in a given time $(1 \mathrm{~h})$ between the $\mathrm{COF}_{\text {sphere }}$ and $\mathrm{COF}_{\text {fiber }}$ films, it becomes clear that, the fiber assembled films follow faster degradation kinetics (Figure S31). For example, in Tp-Azo COF at $200{ }^{\circ} \mathrm{C}$, the Tp-Azo ${ }_{\text {fiber }}$ films undergo a $\sim 0.9$ wt $\%$ mass loss

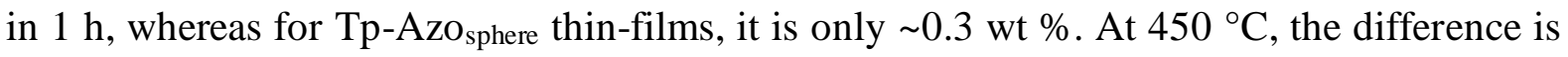

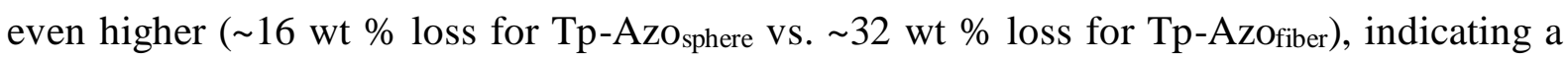
faster degradation for the fiber-based films at a higher temperature. 
Similarly, in Tp-DPP COF at $300{ }^{\circ} \mathrm{C}$, the Tp-DPP fiber films undergo a $\sim 7.5$ wt $\%$ mass loss in $1 \mathrm{~h}$, whereas for $\mathrm{Tp}-\mathrm{DPP}_{\text {sphere }}$ thin-films, it is only $\sim 1.6 \mathrm{wt} \%$. At $350{ }^{\circ} \mathrm{C}$, the difference is

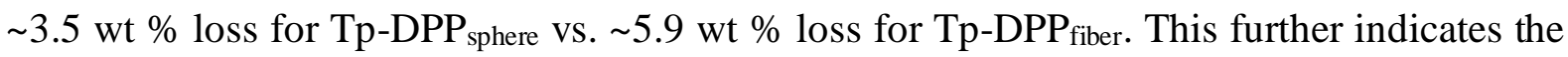
same trend of faster degradation for the fiber-based films.
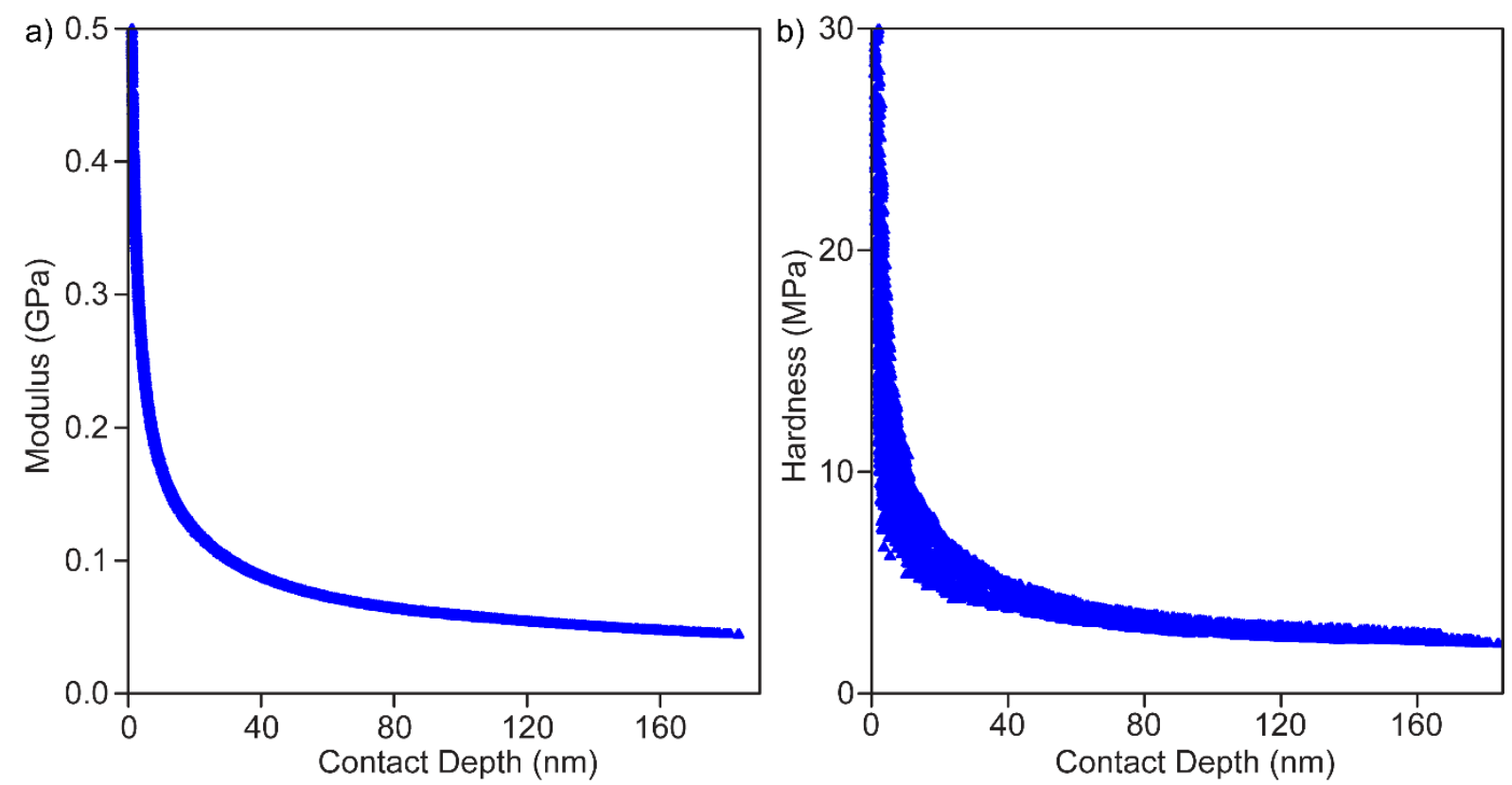

Figure S32: a) Reduced modulus $\left(E_{r}\right)$ vs. contact depth and b) hardness $(H)$ vs. contact depth

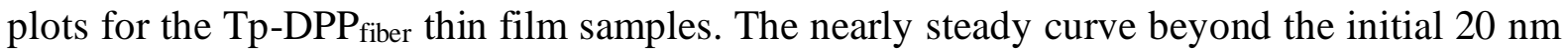
depth reveals that the values obtained are reliable and not affected by substrate effects. 


\section{Section S-12: References}

1. Chong, J. H.; Sauer, M.; Patrick, B. O.; MacLachlan, M. J. Org. Lett. 2003, 5, 38233826.

2. Kiran, M. S. R. N.; Varughese, S.; Reddy, C. M.; Ramamurty, U. Desiraju, G. R. Cryst. Growth Des. 2010, 10, 4650-4655.

3. Gabriele, B. P. A. Williams, C. J.; Lauer, M. E.; Derby, B.; Cruz-Cabeza, A. J. Cryst. Growth Des. 2020, 20, 5956-5966.

4. Wang, Z.; Yu, Q.; Huang, Y.; An, H.; Zhao, Y.; Feng, Y.; Li, X.; Shi, X.; Liang, J.; Pan, F.; Cheng, P.; Chen, Y.; Ma, S.; Zhang, Z. ACS Cent. Sci. 2019, 5, 1352-1359.

5. Hao, Q.; Zhao, C.; Sun, B.; Lu, C.; Liu, J.; Liu, M.; Wan, L.-J.; Wang, D. J. Am. Chem. Soc. 2018, 140, 12152-12158.

6. Miller, K. A.; Alemany, L. B.; Thomas, E. L.; Egap, E. https://doi.org/10.26434/chemrxiv.13100546.v1.

7. Liu, Y.; Ma, Y.; Zhao, Y.; Sun, X.; Gándara, F.; Furukawa, H.; Liu, Z.; Zhu, H.; Zhu, C.; Suenaga, K.; Oleynikov, P.; Alshammari, A. S.; Zhang, X.; Terasaki, O.; Yaghi, O. M. Science 2016, 351, 365.

8. Tan, J. C.; Bennett, T. D.; Cheetham, A. K. PNAS 2010, 107, 9938.

9. Mukherjee, S.; Zeng, Z.; Shirolkar, M. M.; Samanta, P.; Chaudhari, A. K.; Tan, J.-C.; Ghosh, S. K. Chem. Eur. J. 2018, 24, 1 -9.

10. Tian, T.; Zeng, Z.; Vulpe, D.; Casco, M. E.; Divitini, G.; Midgley, P. A.; SilvestreAlbero, J. Nat Mater. 2018, 2, 174-179.

11. Rubin, D. J.; Amini, S.; Zhou, F.; Su, H.; Miserez, A.; Joshi, N. S. ACS Nano 2015, 9, 3360.

12. Yang, L., et al. Journal of Biomedical Materials Research Part A, 2007, 82A, 160168. 
13. Shen, Z.L., et al. Biophysical Journal, 2008, 95, 3956-3963.

14. Knowles, T. P. J.; Buehler, M. J. Nat. Nanotech. 2011, 6, 469-479.

15. Adamcik, J.; Lara, C.; Usov, I.; Jeong, J. S.; Ruggeri, F. S.; Dietler, G.; Lashuel, H. A.; Hamley, I. W.; Mezzenga, R. Nanoscale 2012, 4, 4426-4429. 